\title{
SHP-2 phosphatase contributes to KRAS-driven intestinal oncogenesis but prevents colitis-associated cancer development
}

\author{
Jessica Gagné-Sansfaçon ${ }^{1}$, Geneviève Coulombe ${ }^{1}$, Marie-Josée Langlois ${ }^{1}$, Ariane \\ Langlois $^{1}$, Marilene Paquet ${ }^{2}$, Julie Carrier ${ }^{3}$, Gen-Sheng Feng ${ }^{4}$, Cheng-Kui $\mathbf{Q u}^{5}$, \\ Nathalie Rivard ${ }^{1}$ \\ ${ }^{1}$ Department of Anatomy and Cell Biology, Cancer Research Pavilion, Faculty of Medicine and Health Sciences, Université de \\ Sherbrooke, Sherbrooke, QC, Canada \\ ${ }^{2}$ Département de microbiologie et pathologie, Université de Montréal, St-Hyacinthe, QC, Canada \\ ${ }^{3}$ Department of Medicine, Université de Sherbrooke, Sherbrooke, QC, Canada \\ ${ }^{4}$ Department of Pathology and Division of Biological Sciences, University of California San Diego, La Jolla, CA, USA \\ ${ }^{5}$ Department of Pediatrics, Emory University School of Medicine, Atlanta, GA, USA \\ Correspondence to: Nathalie Rivard, email: Nathalie.Rivard@USherbrooke.ca \\ Keywords: SHP-2, colorectal cancer, colitis-associated cancer, oncogene, mitogen-activated protein kinase \\ Received: May 06, $2016 \quad$ Accepted: August 13, $2016 \quad$ Published: August 25, 2016
}

\section{ABSTRACT}

A major risk factor of developing colorectal cancer (CRC) is the presence of chronic inflammation in the colon. In order to understand how inflammation contributes to CRC development, the present study focused on SHP-2, a tyrosine phosphatase encoded by PTPN11 gene in which polymorphisms have been shown to be markers of colitis susceptibility. Conversely, gain-of-function mutations in PTPN11 gene (E76 residue) have been found in certain sporadic CRC. Results shown herein demonstrate that SHP-2 expression was markedly increased in sporadic human adenomas but not in advanced colorectal tumors. SHP-2 silencing inhibited proliferative, invasive and tumoral properties of both intestinal epithelial cells (IECs) transformed by oncogenic KRAS and of human CRC cells. IEC-specific expression of a SHP-2 ${ }^{\mathrm{E} 76 \mathrm{~K}}$ activated mutant in mice was not sufficient to induce tumorigenesis but markedly promoted tumor growth under the $\boldsymbol{A p c ^ { \mathrm { Min } / + }}$ background. Conversely, mice with a conditional deletion of SHP-2 in IECs developed colitis-associated adenocarcinomas with age, associated with sustained activation of Wnt/ $\beta$-catenin, NFKB and STAT3 signalings in the colonic mucosae. Moreover, SHP-2 epithelial deficiency considerably increased tumor load in $\mathrm{Apc}^{\mathrm{Min} /+}$ mice, shifting tumor incidence toward the colon. Overall, these results reveal that SHP-2 can exert opposing functions in the large intestine: it can promote or inhibit tumorigenesis depending of the inflammatory context.

\section{INTRODUCTION}

The pathogenic mechanisms driving colorectal cancer (CRC) development are complex and heterogeneous. A major component increasing the risk of CRC is the presence of inflammatory bowel diseases (IBD), particularly ulcerative colitis (UC) [1-2]. For both UC-associated CRCs and sporadic CRCs, many mutations occur during the carcinogenic process. The development of sporadic CRC generally involves alterations in the oncogene KRAS and the tumor suppressor genes
APC, SMAD4 and TP53 [3-5]. The pathogenesis of UC-associated CRC appears to differ, involving an orderly progression from inflamed and hyperplastic epithelia to flat dysplasia and lastly to adenocarcinoma. The increased risk to develop CRC in UC patients is presumably attributable to the long-term harmful effects of sustained inflammation in the colon of these patients [1]. One deleterious effect of chronic inflammation is the increased production of reactive oxygen species (ROS) causing oxidative DNA damage [5]. Loss of expression or mutation of the tumor-suppressor gene TP53 is probably 
a key early event in UC-associated carcinogenesis [1-5]. Nevertheless, our knowledge of the underlying cellular mechanisms involved in this process remains incomplete.

Notably, polymorphisms in the PTPN11 gene were previously associated with increased susceptibility to develop UC [6]. PTPN11 gene codes for the Src homology 2-domain containing tyrosine phosphatase (SHP-2) which is ubiquitously expressed and which closely regulates several cell processes including proliferation, differentiation, chemotaxis and survival [7]. Genetic and biochemical evidence demonstrate that SHP-2 positively regulates the RAS/Mitogen-Activated Protein Kinase (MAPK) pathway activation by most receptors [7-10]. SHP-2 binds directly to selected tyrosine kinase receptors or, more often, to scaffolds and becomes activated. Actions of SHP-2 on JAK/STATs $(1,3,5)$ [11], NFאB [9], PI3K/ AKT [12] and RHOA [13] pathways were also reported in various cellular contexts.

To characterize the functional role of SHP-2 in intestinal homeostasis, we have recently generated mice with a deletion of Shp-2 expression specifically in intestinal epithelial cells (IECs). Importantly, these mice (Shp-2 $\left.2^{\mathrm{IEC}-\mathrm{KO}}\right)$ develop spontaneously inflammation in their colon [14]. Chemokine and cytokine secretion profiles indicate that the immunophenotype of Shp$2^{\mathrm{IEC}-\mathrm{KO}}$ mice is similar to patients suffering from UC as opposed to Crohn's disease. Additionally, SHP2 gene transcripts are reduced in intestinal biopsies from patients with active UC, indicating an inverse relationship between SHP-2 expression and intestinal inflammatory phenotype [14]. Of note, the activity of pro-inflammatory transcription factors STAT3 and NF $\kappa$ B are markedly enhanced in SHP-2-deficient colonocytes [14]. Likewise, mice with hepatocytespecific deletion of $S h p-2$ exhibited excessive STAT3 activation and developed severe liver inflammation and tumors with age [15]. Importantly, sustained activation of STAT3 and NFKB are critical for the development of colitis-associated cancer [1,16-17]. Whether loss of epithelial SHP-2 represents an initiating event in colorectal tumorigenesis in the context of chronic inflammation remains however to be determined.

Paradoxically, in humans, gain-of-function mutations of PTPN11 gene have been associated with pediatric leukemias and certain solid carcinomas including hepatocellular carcinoma and CRC [18-19]. These specific mutations increase SHP-2 phosphatase activity and enhance its binding to signaling partners resulting in sustained activation of downstream effectors, particularly the RAS/MAPK pathway [1920]. Importantly, dysregulation of this pathway is also a common event in sporadic colorectal carcinogenesis. Indeed, activating mutations in $K R A S, N R A S$ and $B R A F$ genes are found in up to $60 \%$ of $C R C$ s and are acquired at an early premalignant stage consistent with a role in tumor initiation and/or progression [21-22].
Based on these results, we speculate that SHP-2 can function as an oncoprotein (through the overactivation of the RAS/MAPK pathway) but that under an inflammatory context, it can also act as a tumor suppressor. The present study was therefore designed to elucidate the significance of epithelial SHP-2 in colorectal tumorigenesis.

\section{RESULTS}

\section{SHP-2 expression is increased in early stage colorectal tumors}

SHP-2 mRNA levels were first examined in sporadic human colorectal advanced adenomas and adenocarcinomas at various stages. As illustrated in Figure 1A, transcript levels of SHP-2 were significantly enhanced in colorectal adenomas and stage 1 tumors but not in more advanced stages. SHP-2 protein expression was further analyzed by Western blot to verify if the increased SHP-2 mRNA levels observed in adenomas could be correlated with enhanced protein levels. As shown in Figure 1B, SHP-2 protein levels were also increased in all analyzed human colorectal adenomas compared to normal adjacent tissues. Furthermore, immunohistochemistry analyses demonstrated that the increased expression of SHP-2 was primarily observed in the hyperplastic epithelium and not in the lamina propria (Figure 1C). Thus, these results suggest that increased transcription may contribute to a greater expression of SHP-2 protein in early stages of sporadic CRC.

\section{SHP-2 silencing inhibits proliferative, invasive and tumoral properties of IECs transformed by oncogenic KRAS and human CRC cells}

Approximately $35-40 \%$ of colorectal tumors exhibit mutations in KRAS gene. These mutations occur relatively early in the process of colorectal carcinogenesis [21-22]. Considering the pivotal role of SHP-2 in the activation of the RAS/RAF/MEK/ERK pathway, we examined whether SHP-2 is involved in intestinal epithelial transformation induced by oncogenic KRAS signaling [23-24]. We therefore developed recombinant lentiviruses encoding anti-Shp-2 short hairpin RNA (shRNA) to stably suppress SHP-2 expression in rodent IECs transformed by oncogenic KRAS (IEC-6/KRAS ${ }^{\mathrm{G} 12 \mathrm{~V}}$ cells) [23]. As demonstrated in Figure 2A, levels of SHP-2 protein were markedly diminished in both untransformed and KRAStransformed IEC-6 cells, in contrast to a control shRNA which had no effect. SHP-2 knock-down significantly reduced the proliferation rate of IEC-6/KRAS cells without severely affecting proliferation of control cells (Figure 2B). In addition, SHP-2 silencing partially restored an epithelial morphology in KRAS-transformed cells (Figure 2C). Inhibition of SHP-2 expression also 
decreased the capability of KRAS cells to grow under anchorage-independent conditions (Figure 2D) and to invade Matrigel (Figure 2E). The ability of these cell populations to form tumor in vivo was subsequently assessed in immunodeficient mice. As illustrated in Figure 2F, KRAS cells induced palpable tumors rapidly, 8 days after injection. Of note, SHP-2 silencing clearly attenuated their tumorigenic potential.
SHP-2 contribution was next analyzed in established human CRC cell lines, namely DLD-1 and SW480 which exhibit an activating mutation in the KRAS gene, and in HT-29 cells which have an activating $B R A F$ mutation (Figure $3 \mathrm{~A}$ ). As shown in Figure 3B, cells transfected with siRNA against SHP-2 exhibited reduced capacity to form colonies in soft agarose and to migrate through Matrigel when compared to cells transfected with a control siRNA (Figure 3C).
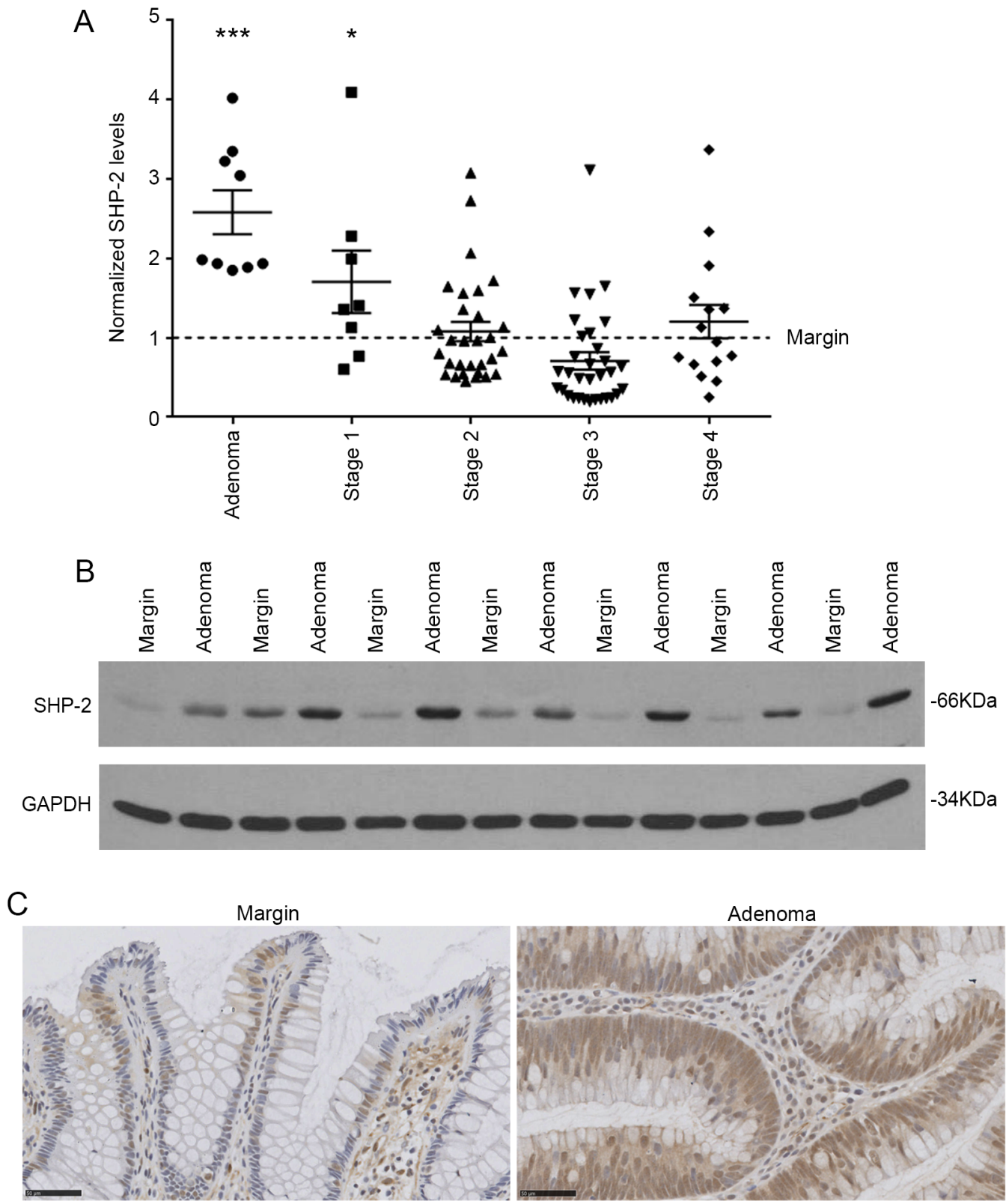

Figure 1: SHP-2 expression in sporadic human colorectal tumors. A. Relative $S H P-2 \mathrm{mRNA}$ levels were determined by q-PCR in human advanced adenomas and adenocarcinomas compared to the paired adjacent healthy tissue (Adenoma N=9; Stage $1 \mathrm{~N}=8$; Stage 2 $\mathrm{N}=30$; Stage $3 \mathrm{~N}=32$; Stage $4 \mathrm{~N}=15$; paired Student's $t$-test). Relative expression was normalized with the housekeeping genes MRPL19, SDHA and YWHAZ. B. Representative immunoblot analysis of SHP-2 protein performed on protein extracts from paired resection margins and adenoma specimens. GAPDH expression is shown as a protein loading control. C. Representative SHP-2 immunohistochemistry performed on human adenomas compared to paired adjacent healthy tissue. Scale bars: $50 \mu \mathrm{M}$. Data are expressed as mean $\pm \mathrm{SEM}$. *p $\leq 0.05$, $* * * p \leq 0.001$. 
A

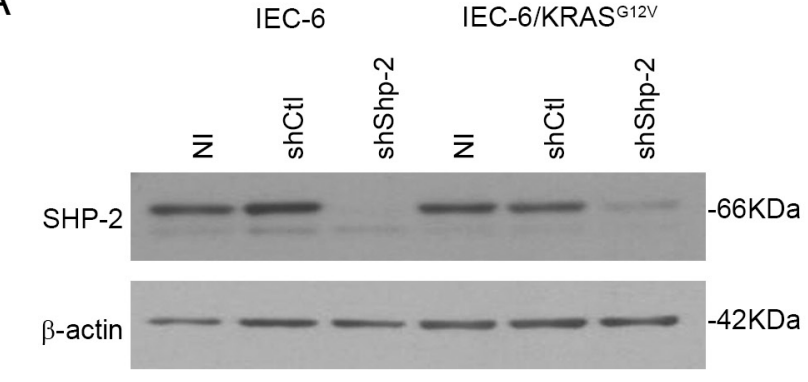

B

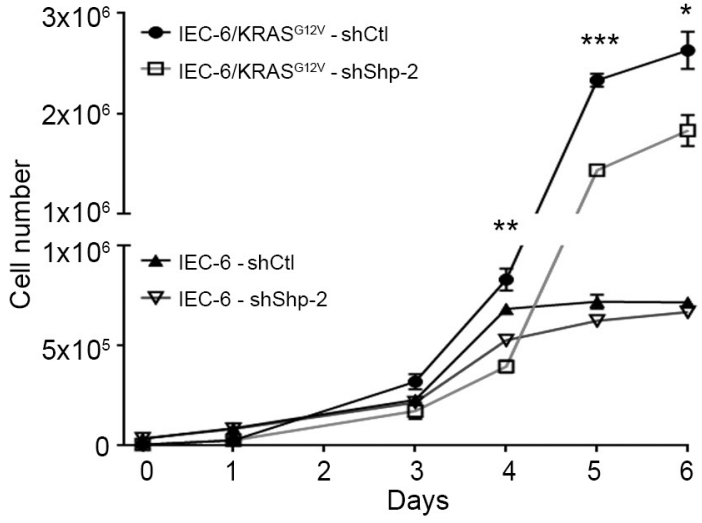

D

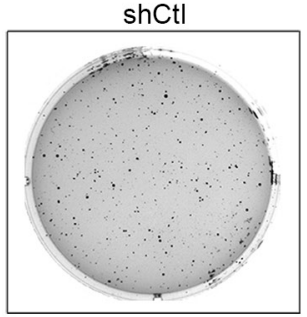

shShp-2
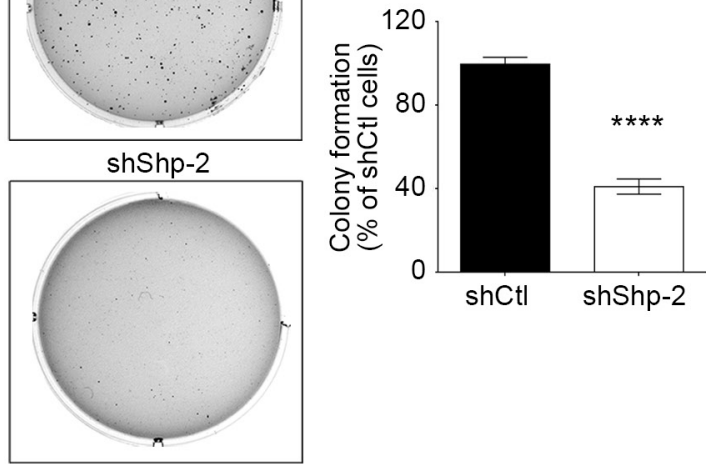

$\mathrm{E}$

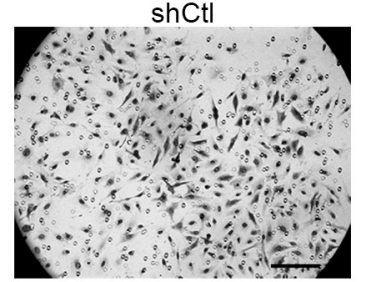

shShp-2

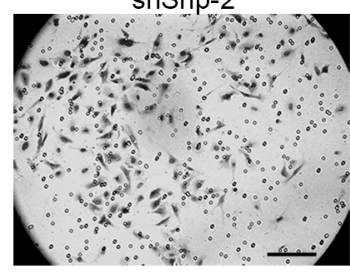

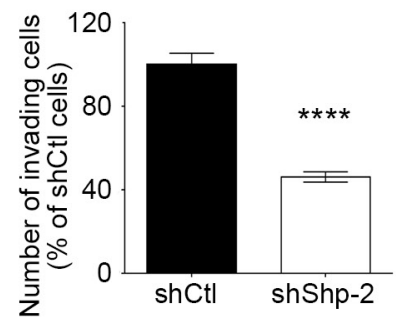

$\mathrm{F}$

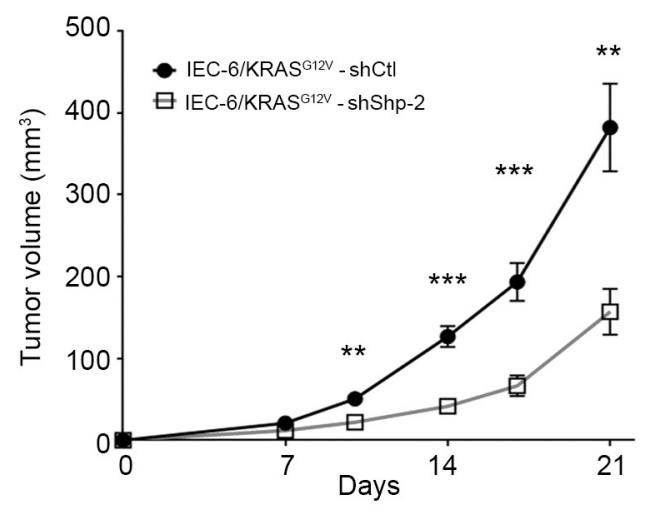

Figure 2: SHP-2 is required for oncogenic activity of $\mathrm{KRAS}^{\mathrm{G} 12 \mathrm{~V}}$ in intestinal epithelial cells. A. Control IEC-6 and $\mathrm{KRAS}^{\mathrm{GL}}$-expressing cells were stably infected with lentiviruses encoding for a control shRNA (scrambled sequence, shCtl) or encoding Shp-2-specific shRNA (shShp-2). After selection, stable cell populations were lysed and protein lysates were analyzed by Western blot for SHP-2 and $\beta$-actin protein expression. NI: *Non-infected. B. Cells were seeded in a 6-well plate, harvested and counted after different time intervals. Growth curves were compared between shCtl and shShp-2 for both control IEC-6 cells and transformed KRAS ${ }^{\mathrm{Gi2V}}$ cells. C. Representative phase-contrast microscopy images of control IEC-6 and KRAS ${ }^{\mathrm{G} 12 \mathrm{~V}}$ cells expressing shShp-2 or shCtl. Bars: $50 \mu \mathrm{m}$. D. IEC-6 KRAS ${ }^{\mathrm{Gl} 2 \mathrm{~V}}$ cells stably expressing shShp-2 or shCtl were cultured in soft agarose for 10 days before 3-(4,5-Dimethylthiazol-2Y1)-2,5-Diphenyltetrazolium Bromide (MTT) staining. E. Invasion capacity of IEC-6 KRAS ${ }^{\text {G12V }}$ cells stably expressing shShp-2 or shCtl was studied using Matrigel-coated Transwells after 48h. The number of cells was determined in 10 fields and the experiments performed in duplicate. F. Tumor growth over time was measured after subcutaneous injection of $5 \times 10^{5}$ IEC-6 KRAS ${ }^{\text {Gi2V }}$ cells stably expressing shShp-2 or shCtl. Results represent mean tumor volume obtained from at least 13 mice injected for each cell line. (A-E) All experiments were conducted on at least three different cell populations (different infections). Data are expressed as mean $\pm \mathrm{SEM}$. ${ }^{*} \mathrm{p} \leq 0.05,{ }^{* *} \mathrm{p} \leq 0.01$, $* * * \mathrm{p} \leq 0.001, * * * * \mathrm{p} \leq 0.0001$. 
SHP-2 is required for full activation of MEK/ ERK signaling in cells expressing oncogenic KRAS

To determine the mechanisms by which SHP-2 silencing interferes with the tumoral properties of cells expressing oncogenic KRAS, transduction pathways known to be associated with these oncogenes were examined, including the MAPK and PI3K/AKT effector pathways [25-26]. As shown in Figure 4A, activation of
MEK $1 / 2$ and ERK1/2 by EGF was severely impaired in IEC-6/KRAS and DLD-1 cells knocked-down for SHP2. By contrast, EGF-induced AKT activation was not significantly affected by SHP-2 silencing (Figure 4B).

Such reduced MEK phosphorylation observed in SHP-2-depleted cells suggests that SHP-2 silencing may interfere with activation of RAS or RAF. Interaction of RAF-1 with RAS has been shown to relieve RAF-1 autoinhibition and is correlated with dephosphorylation of Ser259 and release of 14-3-3 inhibitory proteins [27].
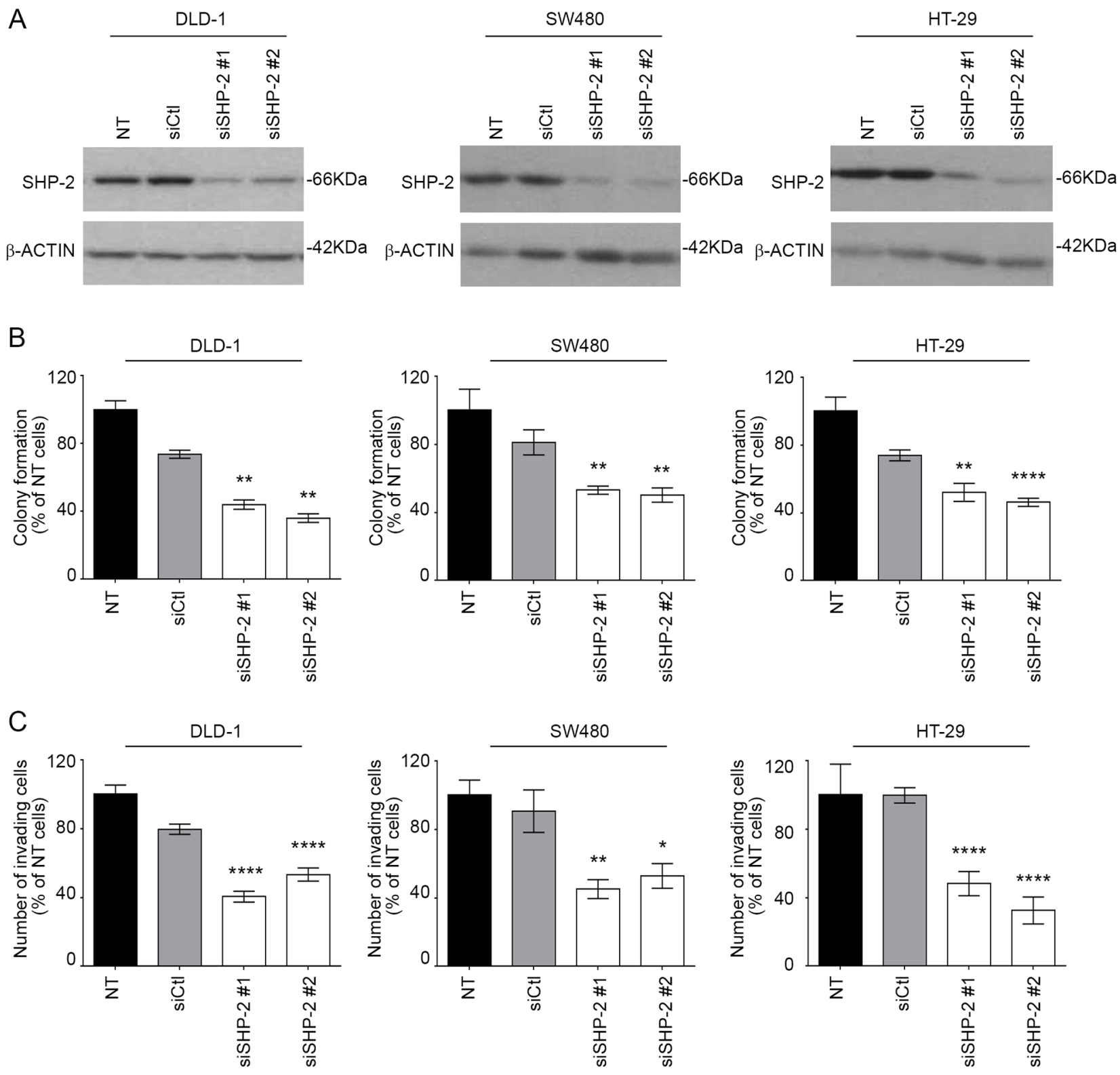

Figure 3: SHP-2 silencing in human CRC cells inhibits growth in soft agar and invasion capacity. A. CRC cells were transfected or not (NT) with a control siRNA (siCtl) or encoding SHP-2-specific siRNAs (siSHP-2\#1 and siSHP-2\#2). Cell populations were lysed after $48 \mathrm{~h}$ and protein lysates were analyzed by Western blotting for SHP-2 and $\beta$-actin expression. B. The capacity of cancer cells to grow in soft agar was assessed following SHP-2 silencing and the number of formed colonies counted after 7 to 14 days with DLD1, SW480 and HT29 cells. C. Invasion capacity was studied using Matrigel-coated Transwells after 48h post-seeding. The number of cells was determined in 10 fields and the experiments performed in duplicate. All experiments were conducted on at least three different cell populations. Data are expressed as mean \pm SEM. ${ }^{*} \mathrm{p} \leq 0.05,{ }^{*} \mathrm{p} \leq 0.01, * * * * \mathrm{p} \leq 0.0001$. 
Additionally, Ser338 and Tyr341 phosphorylation in the SSYY submotif is required for full activity of RAF-1 [27]. As seen in Figure 4C, increased phosphorylation of Ser259 was found in SHP-2 deficient cells. Of note, decreased phosphorylation of Ser338 was noticed in SHP-2-depleted cells while phosphorylation of Tyr340/341 was enhanced (Figure 4C). Since tyrosine phosphorylation of RAF proteins alone is not sufficient to activate the RAF kinase [28], our data strongly suggest that RAF-1 activation is likely impaired in SHP-2 deficient cells.

\section{SHP-2 ${ }^{\mathrm{E} 76 \mathrm{~K}}$ mutant does not act as a classical oncogene but is a novel modifier of intestinal adenoma formation}

Activating mutation in PTPN11 gene (E76G) has been observed in certain CRCs $[18,29]$.To investigate the impact of somatic mutation on E76 residue in vivo,
Ptpn11 ${ }^{\text {E76Kneo/+/Villin-Cre }} e^{+}$mice (Shp-2 $2^{\text {IEC-E76K }}$ mice) were generated by crossing Ptpn $11^{\text {E76Kneo/+ }}$ mice with Villin-Cre mice that express Cre in IECs. Neo deletion efficiency in IECs of these mice was almost complete and SHP-2 ${ }^{\mathrm{E} 76 \mathrm{~K}}$ expression was similar to that of wild-type SHP-2 in Ptpn $11^{+/+} /$Villin-Cre $e^{+}$mice (data not shown). As illustrated in Figure 5A, IEC-specific expression of SHP-2 ${ }^{\mathrm{E} 76 \mathrm{~K}}$ mutant did not severely alter colonic architecture, except for a significant increase in crypt depth and proliferation (Figure 5A). Importantly, no change in colon phenotype of these mice was observed after 1 year, indicating that expression of activating SHP-2 mutation in IECs is not sufficient to induce their oncogenic transformation (Figure 5A). Shp-2 ${ }^{\mathrm{IEC}-\mathrm{E} 76 \mathrm{~K}}$ mutant mice were then subsequently crossed with $A p c^{M i n /+}$ mice, heterozygous for an $A p c$ truncation mutation frequently found in human sporadic CRC, which spontaneously develop adenomas in the intestine [30]. Of note, $A p c^{M i n / t}$ mice in C57BL/6
A

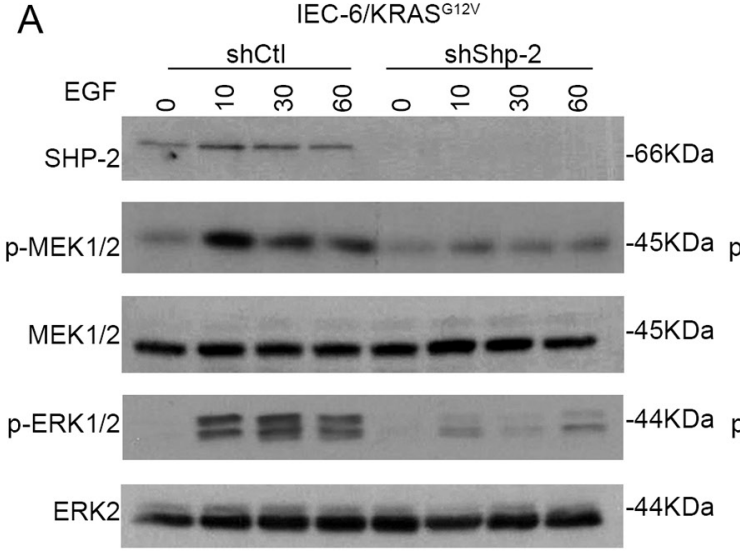

B

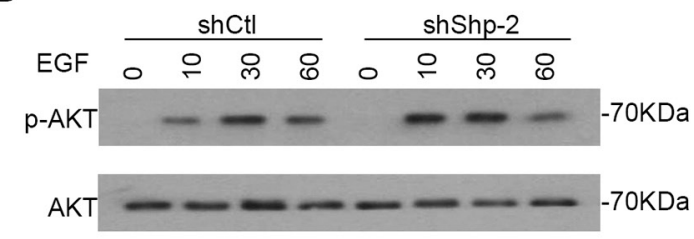

C

IEC-6/KRASG12V

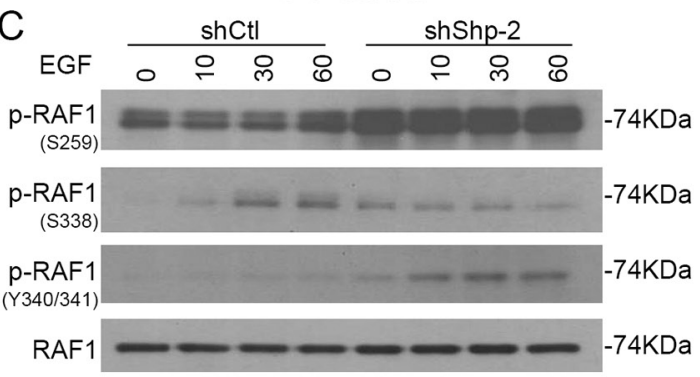

DLD-1
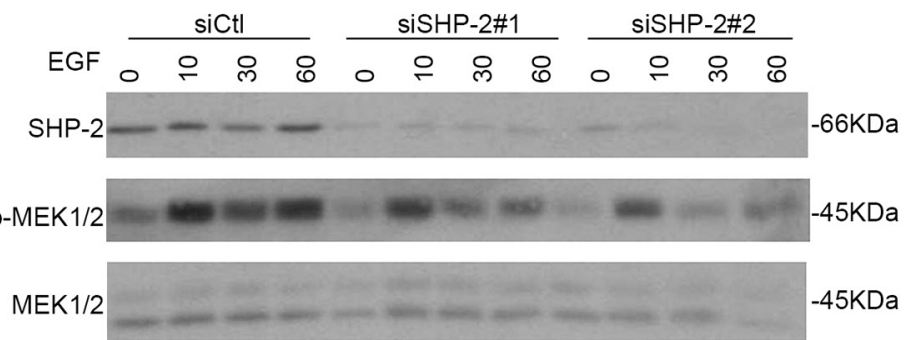

p-ERK1/2

ERK2

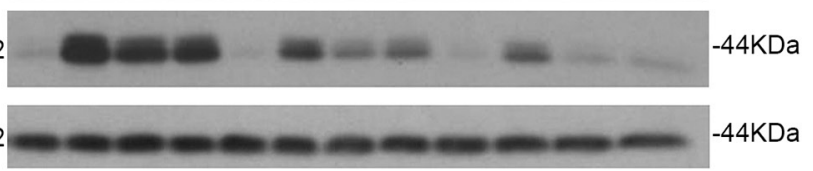

DLD-1

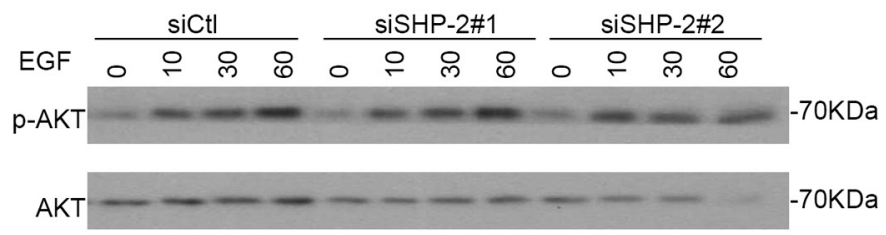

DLD-1

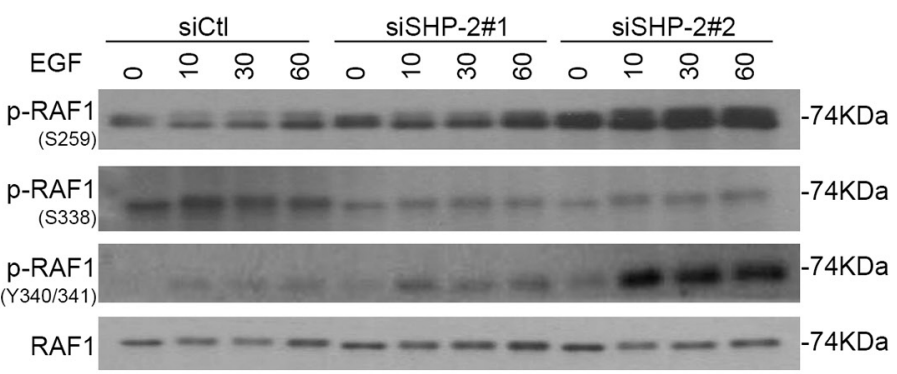

Figure 4: SHP-2 is required for full activation of MEK/ERK signaling in cells expressing oncogenic KRAS. KRAS ${ }^{\mathrm{G} 12 \mathrm{~V}}$ IEC-6 cells and DLD-1 CRC cells in which SHP-2 was downregulated or not by RNA silencing were serum-starved during 24h and thereafter stimulated with $100 \mathrm{ng} / \mathrm{ml} \mathrm{EGF}$ for 10,30 and $60 \mathrm{~min}$. Cells were harvested and phosphorylation levels of MEK1/2 (S217/S221), ERK1/2 (T202/Y204) A. AKT (S473) B. and RAF-1 (S259/S338/Y340/Y341) C. were determined by Western blotting. All experiments were conducted on at least three different cell populations and representative immunoblots are shown. 
A
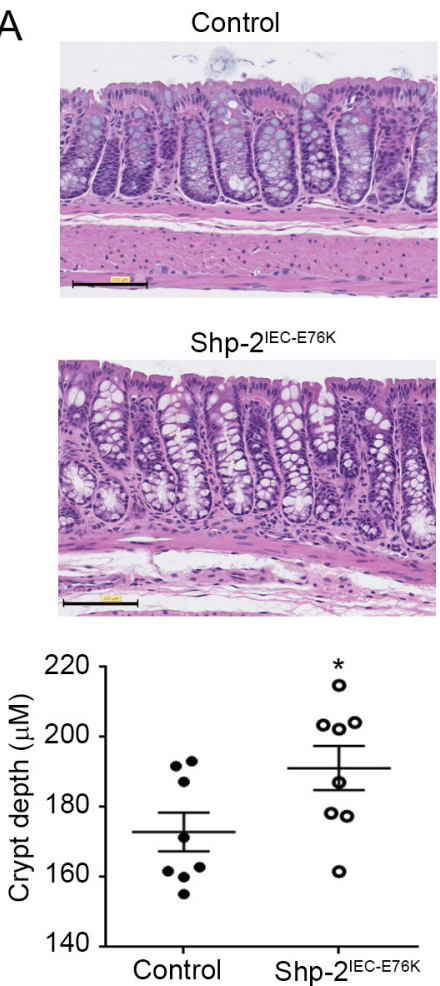

Control

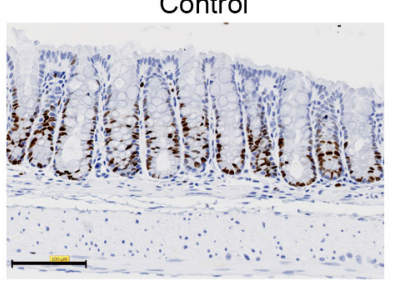

Shp-2 $2^{\mathrm{IEC}-\mathrm{E} 76 \mathrm{~K}}$
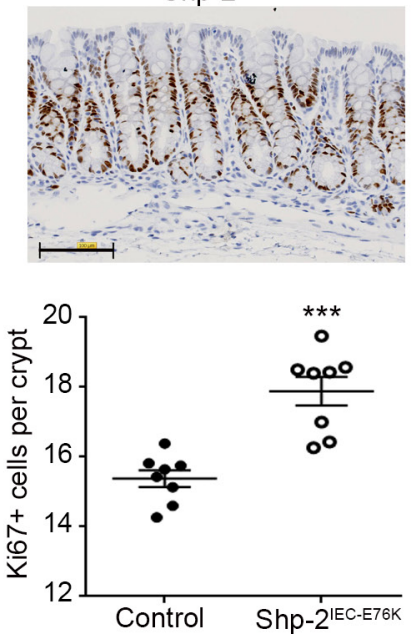

B
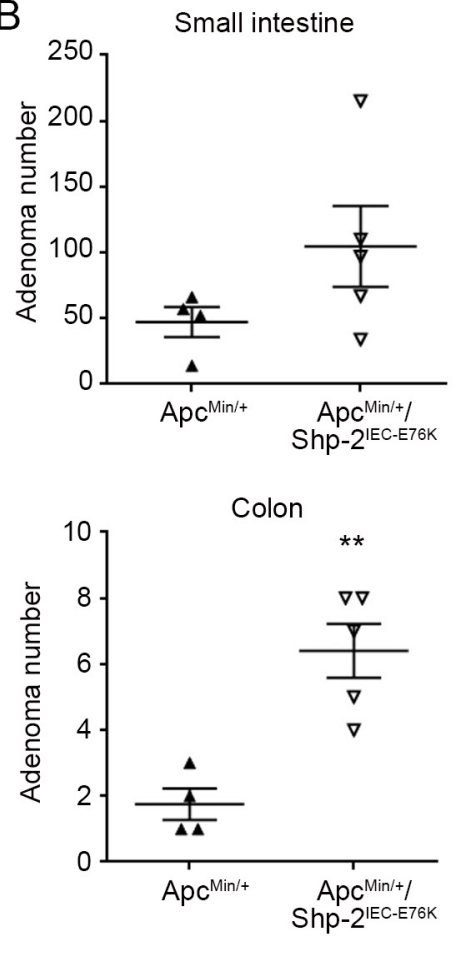

$\mathrm{Apc}^{\mathrm{Min} /+} / \mathrm{Shp}-2^{\mathrm{IEC}-\mathrm{E} 76 \mathrm{~K}}$
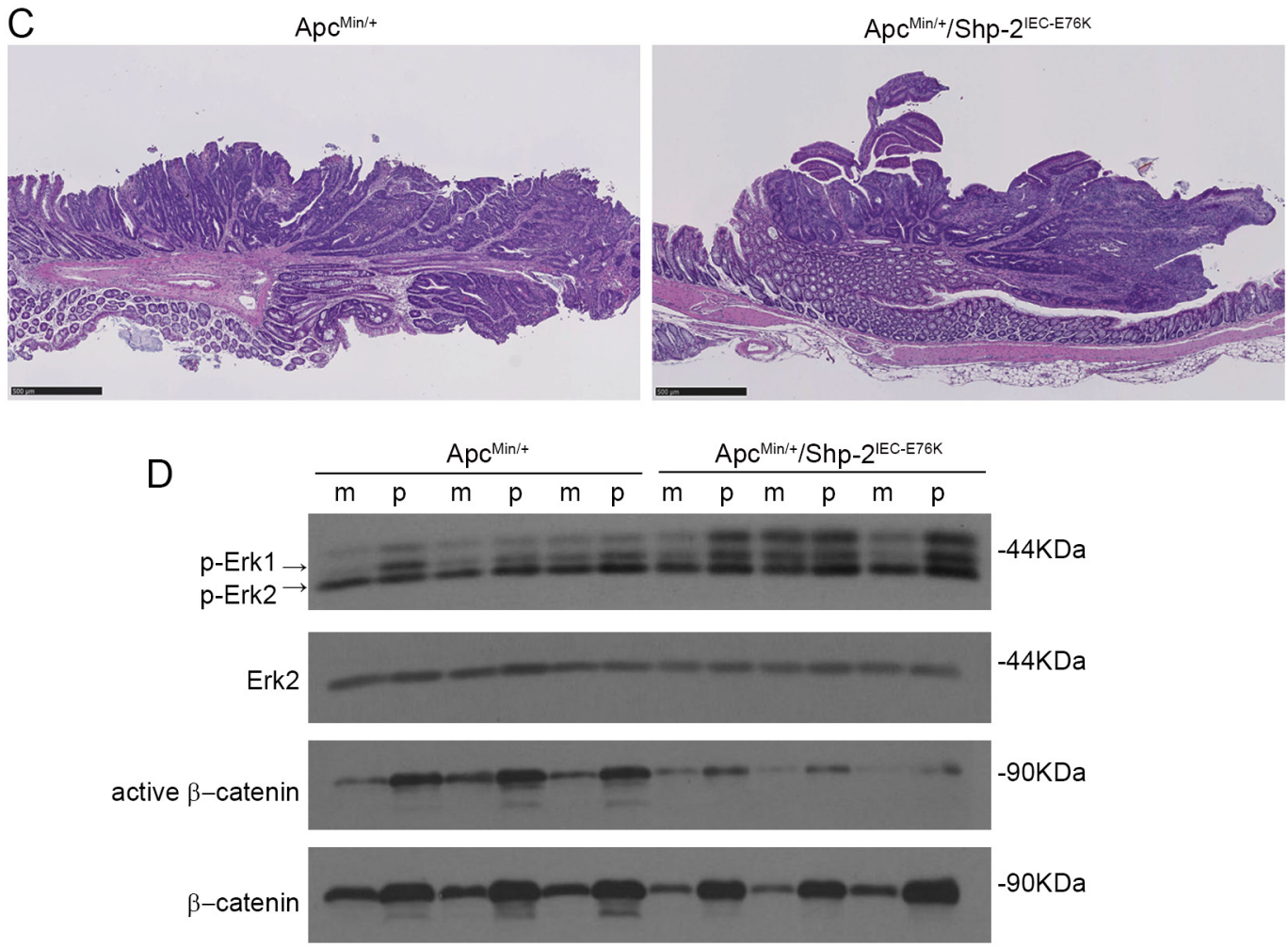

Figure 5: Expression of activated SHP-2 ${ }^{\mathrm{E} 76 \mathrm{~K}}$ mutant in intestinal epithelium deregulates crypt proliferation and increases tumor multiplicity in $\mathrm{Apc}^{\mathrm{Min/} /}$ mice. A. Colonic sections obtained from 15 month-old control and Shp-2IEC-E76K mice were stained with H\&E for histological analysis and KI67 for proliferation determination $(\mathrm{N}=8)$. Scale bars: $100 \mu \mathrm{m}$. B. The number of polyps formed in the small and large intestines was determined at 3 months of age in both $A p c^{\text {Min }+}$ and $A p c^{\text {Min/+ }}$ mice expressing activated SHP-2 mutant $\left(A p c^{\text {Min'+; }}\right.$

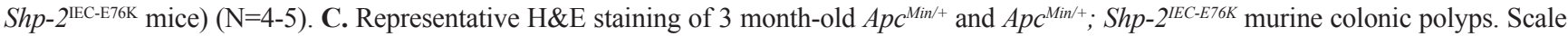
bars: $500 \mu \mathrm{m}$. D. Western blot analyses were performed on paired specimens of margins (m) and polyps (p) extracted from $A p c^{M i n /+}$ and $A p c^{M i n /+}$; Shp-2IECE76KK mice. Levels of total and phosphorylated ERK1/2 (T202/Y204) and total and non-phospho (active) $\beta$-catenin were specifically analyzed. Representative immunoblots are shown. Data are expressed as mean \pm SEM. $* \mathrm{p} \leq 0.05,{ }^{*} \mathrm{p} \leq 0.01, * * * \mathrm{p} \leq 0.001$. 
background have been shown to die before they reach four months due to anemia and intestinal obstruction resulting from polyposis [30]. Therefore, we sacrificed the mice at three months of age. Of note, the survival rate was similar between $A p c^{\mathrm{Min/+} ;} \mathrm{Shp}-2^{\mathrm{IEC-E76K}}$ and $\mathrm{Ap} \mathrm{c}^{\mathrm{Min} /+}$ mice at this age. As shown in Figure $5 \mathrm{~B}$, we observed that SHP-2 ${ }^{\mathrm{E} 76 \mathrm{~K}}$ expression in $A p c^{\mathrm{Min} /+}$ mice significantly increased tumor multiplicity in the small and large intestines (Figure 5B). Histologically, both $A p c^{\mathrm{Min} /+}$ and $A p c^{M i n /+} ; S h p-2^{I E C-E 76 K}$ mice developed typical intestinal intraepithelial neoplasia (GIN) and adenomas (Figure 5C). As expected, polyps from $A p c^{\mathrm{Min} /+}$ mice exhibited increased accumulation of $\beta$-catenin protein confirming the deregulation of APC signaling in these tumors (Figure 5D). Accordingly, levels of active (non-phosphorylated) $\beta$-catenin were significantly enhanced in polyps of $A p c^{\mathrm{Min} /+}$ mice in comparison to normal tissues. Reduced levels of total and active $\beta$-catenin were however found in colonic epithelium and polyps from $A p c^{\mathrm{Min} /+} ; \mathrm{Shp}-2^{\mathrm{IEC}-}$ ${ }^{E 76 K}$ mice suggesting an attenuation of the $\mathrm{Wnt} / \beta$-catenin signaling in these double mutant mice. Hence, pathways distinct of $\mathrm{Wnt} / \beta$-catenin signaling most likely triggered these changes in colon tumor multiplicity. SinceMEK/ ERK signaling activation has been deemedfundamental for intestinal tumor development in $\mathrm{Apc}^{\mathrm{Min} /+}$ mice [31], immunoblot analyses were performed to elucidate whether changes in ERK phosphorylation status can be observed in colon epithelial samples and polyps after expression of the activated SHP-2 ${ }^{\mathrm{E} 76 \mathrm{~K}}$ mutant. Analyses revealed that levels of p-ERK1/2 were indeed higher in colon epithelium and polyps of $A p c^{M i n /+} ; S h p-2^{I E C-E 76 K}$ mice than in epithelium and polyps of control $A p c^{\mathrm{Min} /+}$ mice.

\section{Shp-2 ${ }^{\text {IEC-KO }}$ mice develop colorectal adenocarcinomas with age}

Persistent inflammation in the colon predisposes patients to $\mathrm{CRC}$. Importantly, we previously reported that SHP-2 deletion in murine IECs (Shp-2 $2^{I E C-K O}$ mice) spontaneously leads to chronic inflammation in the colon, four weeks after birth [14]. Herein, Shp-2 $2^{I E C-K O}$ mice were followed longitudinally to evaluate their phenotype 15 months after birth. As shown in Figure 6, Shp- $2^{I E C-K O}$ mice still exhibited chronic colitis as visualized by the sustained elevated DAI (Figure 6A) and fibrosis (Figure 6B). This persistent inflammation further prompted to investigate whether Shp-2 ${ }^{I E C-K O}$ mice had developed colorectal tumors with age. As shown in Figure 6C, 15 months after birth, Shp-2IEC-KO mice had developed severe dysplasia and infiltrating adenocarcinomas in their distal colon. Some of these mice also developed invasive carcinomas as witnessed by invasion of tumor cells through the lamina propria (Figure 6C, panels 4 and 8, arrows).

To understand the molecular mechanisms involved in tumor development in aged $S h p-2^{I E C-K O}$ mice, we analyzed the activation levels of signaling effectors known to be regulated by SHP-2 and to be involved in chronic intestinal inflammation. As illustrated in Figure 7A, the downstream transcription factors STAT3 and p65NFkB, both of which are tumor-promoting factors, were clearly phosphorylated and activated in the colons of Shp-2 $2^{I E C-K O}$ mice. Immunofluorescence confirmed increased STAT3 phosphorylation in the colon from these mice, especially in epithelial cells (Figure 7B, see arrows). Interestingly, while the expression of acetylated p53 was barely detectable, levels of mutant p53 (R273H) were clearly increased in Shp-2-deficient colons (Figure 7A), indicating that Tp53 gene was targeted during colitis-induced carcinogenesis. Moreover, phosphorylation levels of ERK1/2 MAPK were reduced in aged $S h p-2 \mathrm{KO}$ mice (Figure 7A) as previously observed in younger mice [14]. By contrast, expression levels of $\beta$-catenin protein (Figure 7A) as well as $C d 44, C y c l i n D 1$ and $C-M y c$ transcripts (Figure 7C) were enhanced in the colon of mutant mice suggesting the activation of Wnt/ $\beta$-catenin signaling. Lastly, we analyzed the expression of proinflammatory cytokines IL-6, IL-17 and IL-23 recently shown to influence the development and growth of colitis-associated cancer (CAC) [32-35]. As shown in Figure 7D, levels of these cytokines were indeed significantly increased in aged $S h p-2^{I E C-K O}$ mice.

Immunohistochemistry analysis furthermore demonstrated increased KI67 staining, a marker of proliferation, especially in the nucleus of most epithelial and mesenchymal cells in dysplastic regions of aged Shp$2^{I E C-K O}$ mice while its expression was restricted to the nucleus of proliferative progenitor cells along the colonic crypt in control mice (Figure 8A). Increased phosphoH2AX staining (Figure 8B) as well as Nox 1, Ho-1, iNos and Cox 2 expression (Figure $8 \mathrm{C}$ ) were moreover observed in the colon of 15 month-old Shp-2 $2^{I E C-K O}$ mice indicating the presence of DNA damage and oxidative stress, two typical features of CAC [36-37]. Overall, these results suggest that SHP-2 protects the colonic epithelium against inflammation-induced cell proliferation and DNA damage.

\section{IEC-specific Shp-2 ablation exacerbates tumorigenesis in $\mathrm{Apc}^{\mathrm{Min} /+}$ mice}

To further analyze the suppressive function of SHP2 in inflammation-induced intestinal tumorigenesis, the effect of SHP-2 removal was assessed in $\mathrm{Apc}^{\mathrm{Min} /+}$ mice. As illustrated in Figure 9A, the multiplicity of polyps after SHP-2 epithelial loss was much more dramatic in the colon than in the small intestine of $A p c^{\mathrm{Min} /+} ; \mathrm{Shp}-2^{I E C-K O}$ mice comparatively to control $A p c^{\mathrm{Min} /+}$ mice at three months of age. Importantly, $38 \%$ of $A p c^{M i n+} ; S h p-2^{I E C-K O}$ mice died before three months, probably because of malnutrition caused by severe diarrhea and rectal bledding. Indeed, at this age, Shp-2 $2^{I E C-K O}$ and $A p c^{M i n /+;} S h p-2^{I E C-K O}$ mice have clearly developed chronic colitis as demonstrated by higher DAI in comparison to control littermates (Figure 9B). Histological analysis confirmed the increased 
A

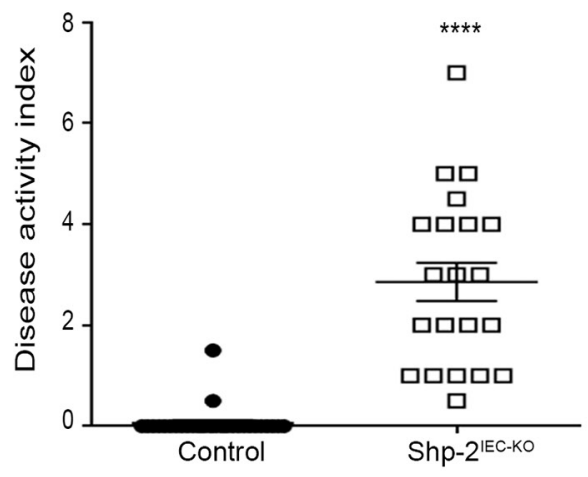

B

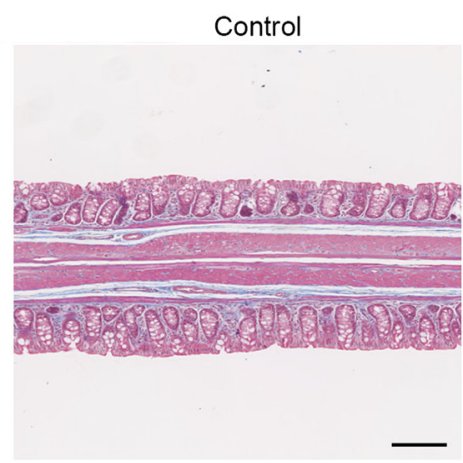

Shp-2 $2^{\mathrm{IEC}-\mathrm{KO}}$

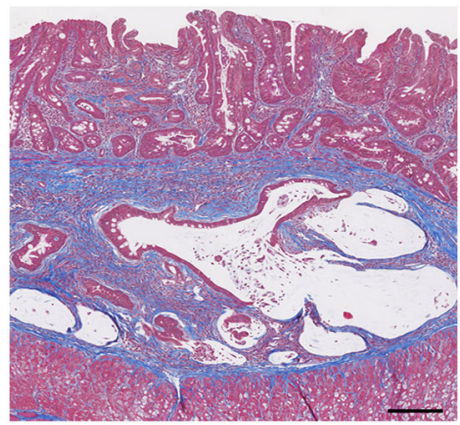

C

1.

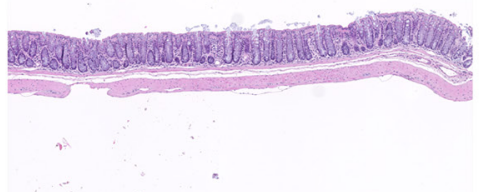

3.

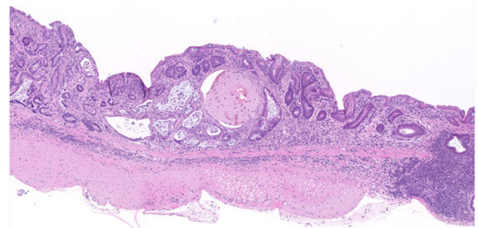

Shp-21EC-Ko

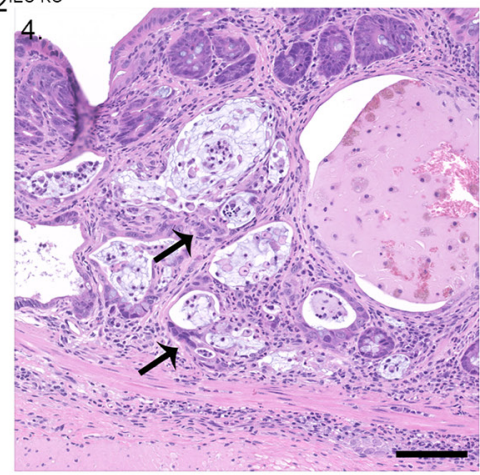

5.
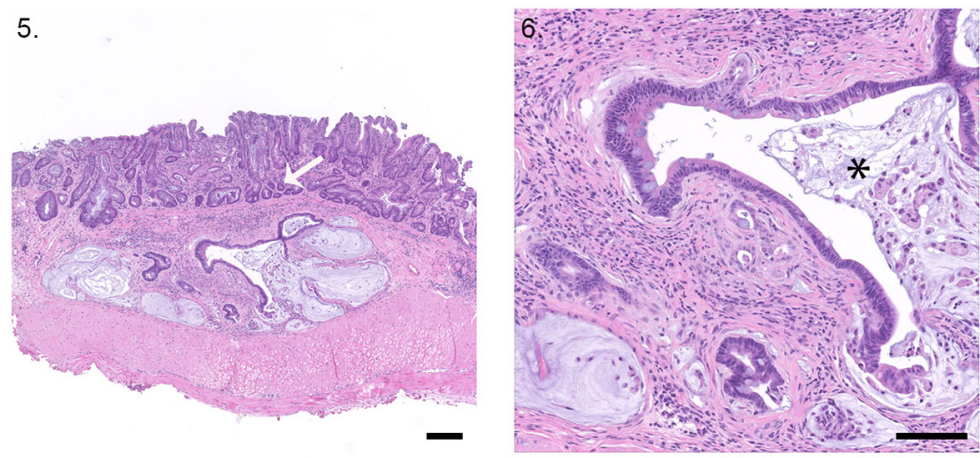

7.
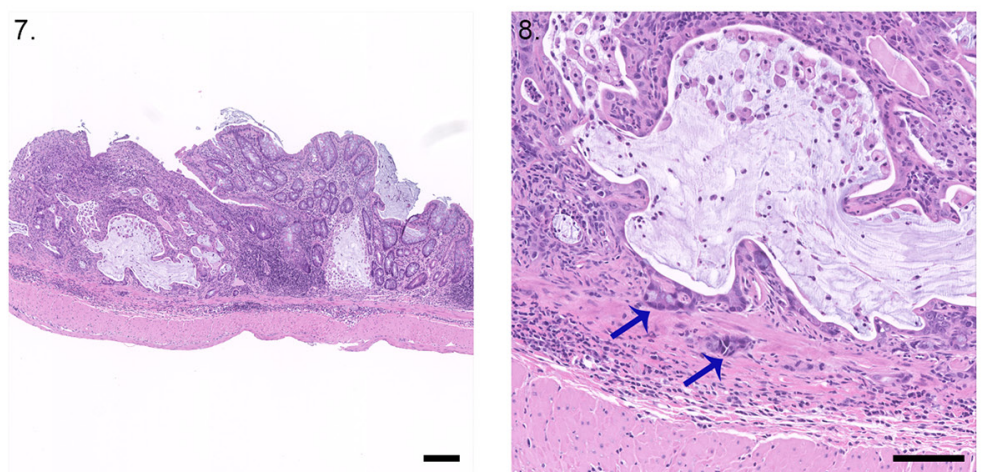

Figure 6: Mice with loss of epithelial SHP-2 expression develop colorectal adenocarcinomas with age. A. Disease activity index of 15-month-old Shp-2 ${ }^{I E C-K O}$ mice and control littermates were calculated by scoring stool softness, occult fecal blood, rectal bleeding and colon rigidity $(\mathrm{N}=20)$. B. Masson's trichrome staining was performed to assess fibrosis in aged $S h p-2^{I E C-K O}$ mice and control littermates $\left(\mathrm{N}=6\right.$; blue=collagen fiber accumulation). C. Representative images of H\&E staining of 15-month-old control and Shp-2 ${ }^{\mathrm{IEC}-\mathrm{KO}}$ murine colons ( $\mathrm{N}=9 ; 100 \%$ of $S h p-2^{I E C-K O}$ mice had developed adenocarcinoma). Blue arrow shows cancerous cells invading the muscularis; black arrow shows cancerous cells in the mucosa; white arrow shows dysplasia; * indicates crypt abscess. Scale bars: $100 \mu \mathrm{m}$. Data are expressed as mean \pm SEM. $* * * * p \leq 0.0001$. 
immune cell infiltration, the reduction in goblet cells and the presence of crypt abscesses in Shp-2-deficient colons (Figure 9C). Importantly, these signs of inflammation were also manifest in the colon of $\mathrm{Apc}^{\mathrm{Min} /+} ; \mathrm{Shp}-2^{I E C-K O}$ mice (Figure 9C), correlating with elevated DAI similar to $S h p-2^{I E C-K O}$ mice (Figure 9B).

In comparison to their corresponding benign epithelium, polyps from $A p c^{\mathrm{Min} /+}$ mice displayed consistently higher phosphorylation levels of ERK1/2, STAT3 and p65RELA (Figure 9D). Importantly, upon deletion of SHP-2 in $\mathrm{Apc}^{\mathrm{Min} /+}$ mice, phosphorylated levels of ERK1/2 were found to be diminished both in the polyps and margins in comparison to $A p c^{\mathrm{Min} /+}$ mice. By contrast, total and active levels of $\beta$-catenin, STAT3 and p65RELA were further increased in the colon of $\mathrm{Apc}^{\mathrm{Min} /+} ; \mathrm{Shp}-2^{\text {IEC-KO }}$ mice. These observations suggest that SHP-2 may exert a tumor suppressive function under an inflammatory setting.

\section{DISCUSSION}

Genetic and biochemical evidence strongly demonstrates that SHP-2 is essential for full activation of RAS/ERK MAPK pathway by most receptors [3839]. Furthermore, activating mutations in PTPN11 gene have also been described in the developmental disorder Noonan syndrome and in pediatric leukemias [40]. Likewise, somatic PTPN11 mutations were found in certain solid tumors such as lung, liver and CRCs [18]. These studies therefore suggest that SHP-2 may be a bona fide oncoprotein [19].

Consistent with the above, SHP-2 was found to be overexpressed in gastric cancer, breast cancer and nonsmall cell lung cancer (NSCLC) [40-42]. However, there have been sparse reports regarding SHP-2 expression in CRC [43]. Recently, Cai et al. (2014) analyzed

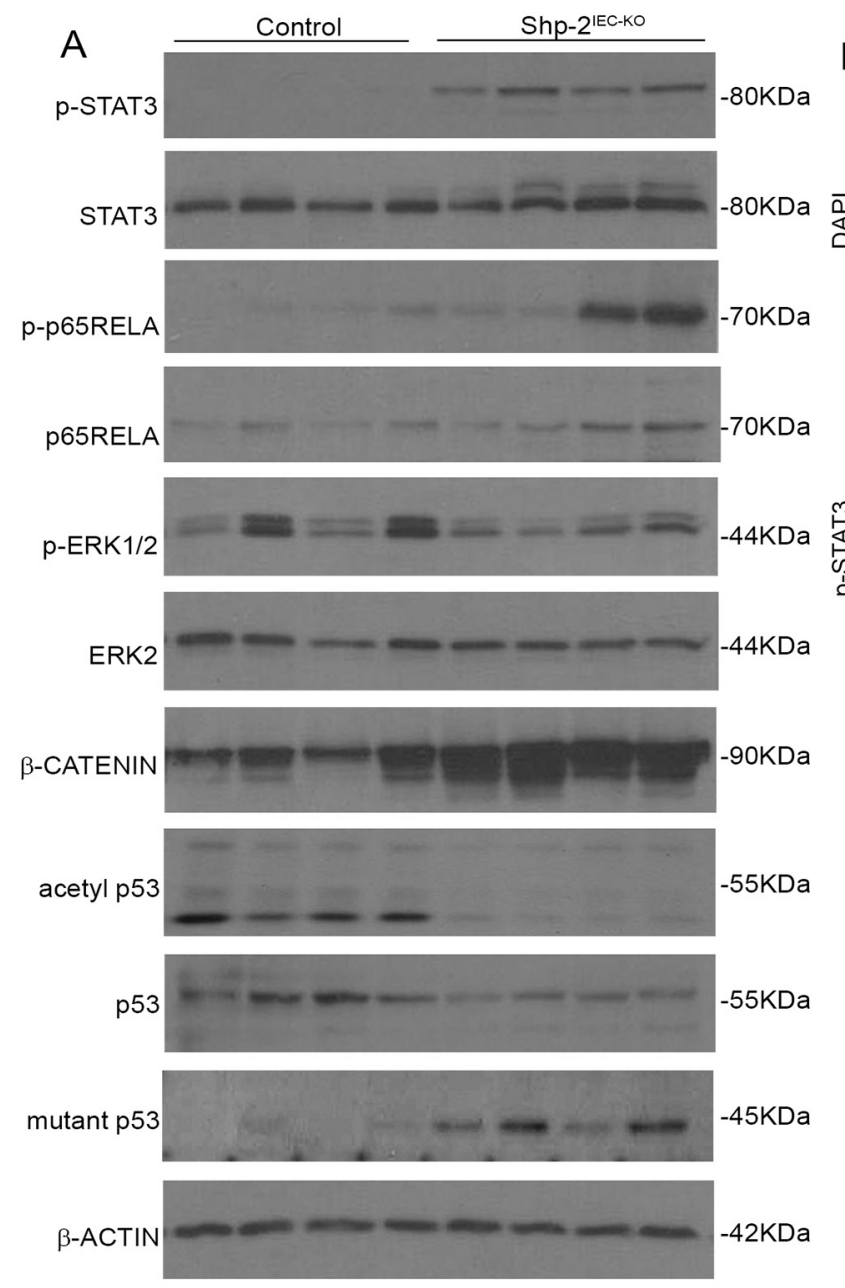

Figure 7: Aged $S h p-2^{I E C-K O}$ mice exhibit several typical features of colitis-associated cancer. A. Mucosal enrichments from 15 month-old $S h p-2^{I E C-K O}$ and control murine colons $(\mathrm{N}=8)$ were analyzed by Western blotting for the expression of selected proteins including total and phosphorylated ERK1/2 (T202/Y204), STAT3 (Y705) and p65NFkB (S536) as well as total $\beta$-catenin, total p53, mutant-p53 $(\mathrm{R} 273 \mathrm{H})$ and acetylated p53 (K379). $\beta$-actin served as loading control. Representative immunoblots are shown. B. Immunofluorescence against phosphorylated STAT3 (Y705) was performed on colonic tissue from 15 month-old control and Shp-2IEC-KO mice. White arrows indicate epithelial cells. Scale bars: $100 \mu \mathrm{m}$. (Continued) 

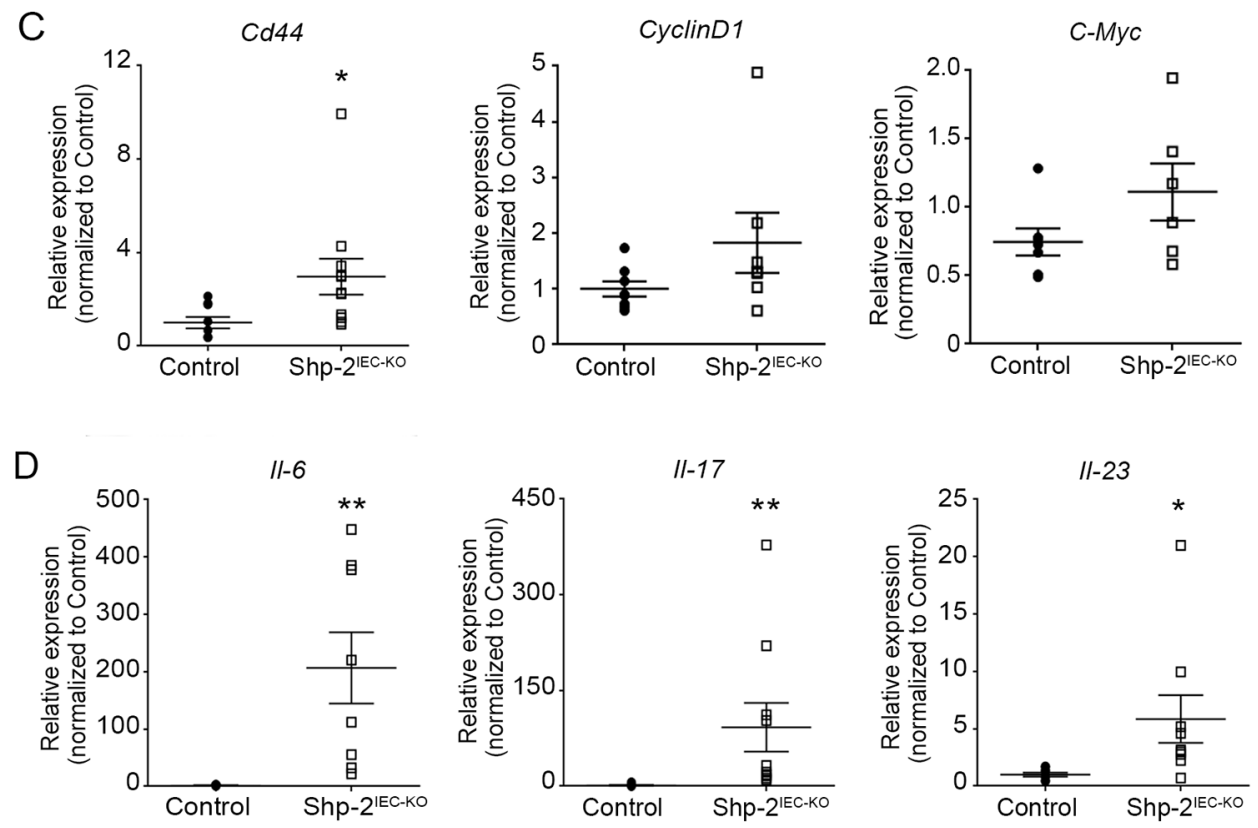

Figure 7: (Continued) Aged Shp-2 ${ }^{I E C-K O}$ mice exhibit several typical features of colitis-associated cancer. C. Quantitative PCR of Cd44, Cyclin D1 ( $\mathrm{p}=0.1385)$ and $C$-Myc ( $\mathrm{p}=0.1254)$ mRNAs in enriched colonic mucosal extracts from 15-month-old control and $S h p-2^{I E C-K O}$ mice were performed. Relative expression was normalized with the housekeeping genes Psmc4, Pum1 and Tbp (N $\geq 6$ ). D. Quantitative PCR of interleukin-6 (IlO), interleukin-17 (Ill7) and interleukin-23 (Il23) mRNAs in enriched colonic mucosal extracts from 15 month-old control and $S h p-2^{I E C-K O}$ mice were performed. Relative expression was normalized with the housekeeping genes $P s m c 4$, Puml and $\operatorname{Tbp}(\mathrm{N} \geq 8)$. Data are expressed as mean $\pm \mathrm{SEM} .{ }^{*} \mathrm{p} \leq 0.05, * * \mathrm{p} \leq 0.01$.

SHP-2 levels in 232 unpaired tumor specimens from patients with CRC. They found that low SHP-2 expression correlated with poor tumor differentiation, late TNM stage and lymph node metastasis. Herein, we found significant increase in SHP-2 mRNA and protein levels in colorectal adenomas and stage 1 tumors. Notably, the increased expression of SHP-2 was primarily detected in the hyperplastic epithelium and not in the lamina propria, indicating that SHP-2 may be involved early in the development of sporadic colorectal tumors. However, in more advanced CRCs, SHP-2 mRNA levels were not significantly modulated in comparison to their corresponding margins. We verified PTPN11 gene promoter methylation in human colon specimens from the TCGA database (TCGA Research Network: http:// cancergenome.nih.gov), interrogated and plotted using the TCGA Wanderer interface [44]. Overall, we did not find significant difference in PTPN11 gene promoter methylation suggesting that it is not the mechanism by which $S H P-2$ gene expression is regulated in advanced CRCs (data not shown). Since the epithelium to stroma ratio varies and changes considerably during tumor progression, one might speculate that this may influence protein/RNA quantification. It would be pertinent in the future to perform immunohistochemistry analyses in advanced tumors to verify SHP-2 expression in epithelial cells versus the surrounding stromal tissue.

Up to $60 \%$ of colorectal tumors exhibit gain-offunction mutations in KRAS, NRAS and BRAF genes
[21-22]. These mutations generally occur early in the adenoma-carcinoma sequence [21], triggering cell transformation, hyperproliferation and anoikis resistance [45-46]. Accordingly, most of colorectal adenomas analyzed herein exhibited $K R A S(\mathrm{G} 12 \mathrm{D}, \mathrm{G} 13 \mathrm{D}, \mathrm{Q} 61 \mathrm{H})$ or $B R A F(\mathrm{~V} 600 \mathrm{E})$ activating mutations, in combination with $A P C$ inactivating mutations (exon 15) [24]. Considering the significance of SHP-2 in the control of RAS pathway, we speculated that this phosphatase likely plays an important role in colorectal carcinogenesis. Indeed, we found that SHP-2silencing in KRAS-transformed IECs severely impaired both their proliferation rate and invasive capabilities. Moreover, cells displaying knocked-down SHP-2 exhibited reduced tumor formation capacity. Similarly, targeting SHP-2 by RNAi in human KRAS or BRAF-mutated established CRC cells limited their ability to grow in soft agar and to invade Matrigel. Interestingly, these inhibitory effects of SHP-2 silencing were associated with reduced phosphorylation of RAF, MEK and ERK proteins in cells expressing oncogenic KRAS. At first glance, these data are in agreement with those demonstrating that the phosphatase activity of SHP2 is indeed necessary for full RAS activation. Accordingly, cells expressing dominant-negative SHP-2 [38] or Ptpn11 gene exon 3-deleted mouse embryonic fibroblasts [39] exhibit defective RAS activation. Furthermore, SHP-2 may also function either downstream and/or in parallel to RAS since other data have shown that SHP-2 inhibition can impair the activation of downstream effectors 
A

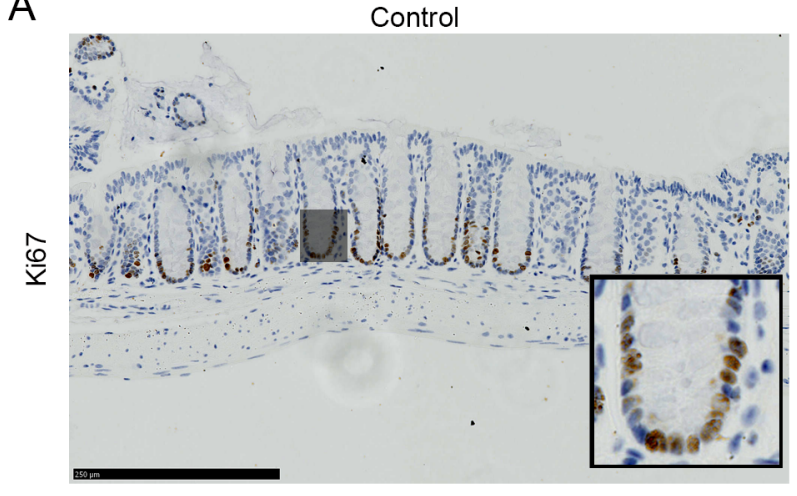

B

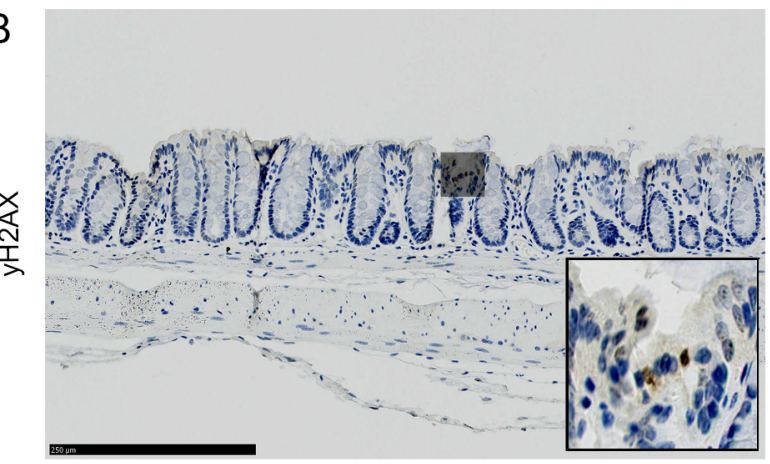

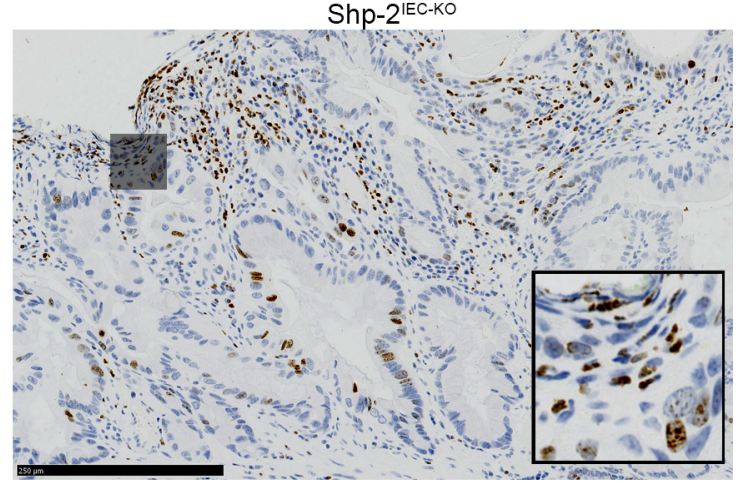

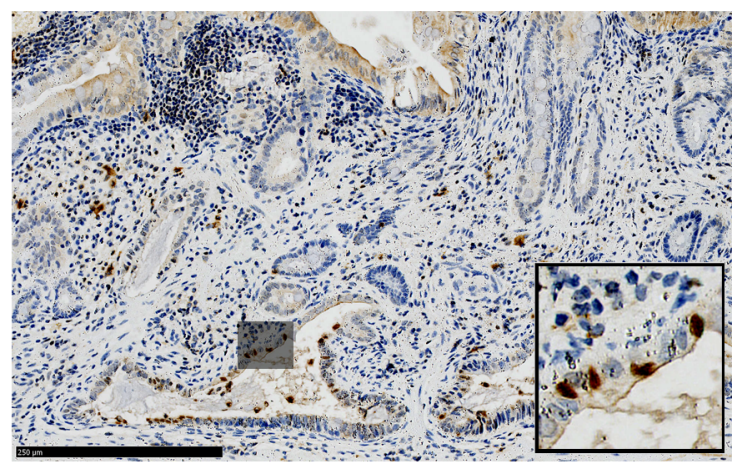

Ho-1

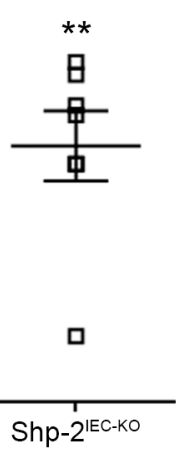

Cox-2

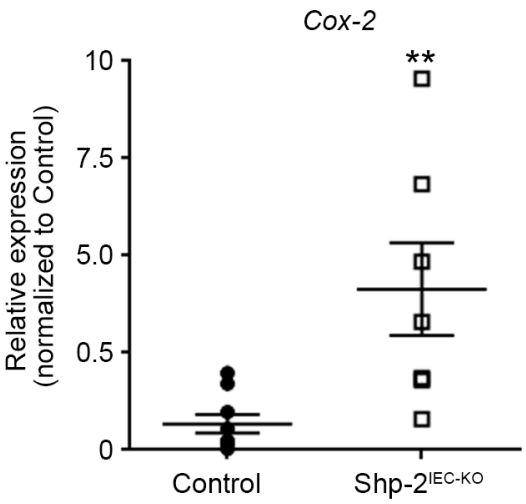

Figure 8: Aged $S h p-2^{I E C-K O}$ mice exhibit increased epithelial proliferation and signs of oxidative stress in their colon. (A-B) Representative images of 15 month-old control and Shp-2 ${ }^{I E C-K O}$ murine colons stained for KI67 (A) and phospho-H2AX (B) are shown (representative of N=6). Scale bars: $250 \mu \mathrm{m}$. C. Quantitative PCR of Nox1, Ho-1, iNos and Cox-2 mRNAs in enriched colonic mucosal extracts from 15-month-old control and $S h p-2^{I E C-K O}$ mice were performed. Relative expression was normalized with the housekeeping genes Psmc4, Pum 1 and Tbp (N=9). Date are represented as mean \pm SEM. ${ }^{*} \mathrm{p} \leq 0.05, * * \mathrm{p} \leq 0.01$. 

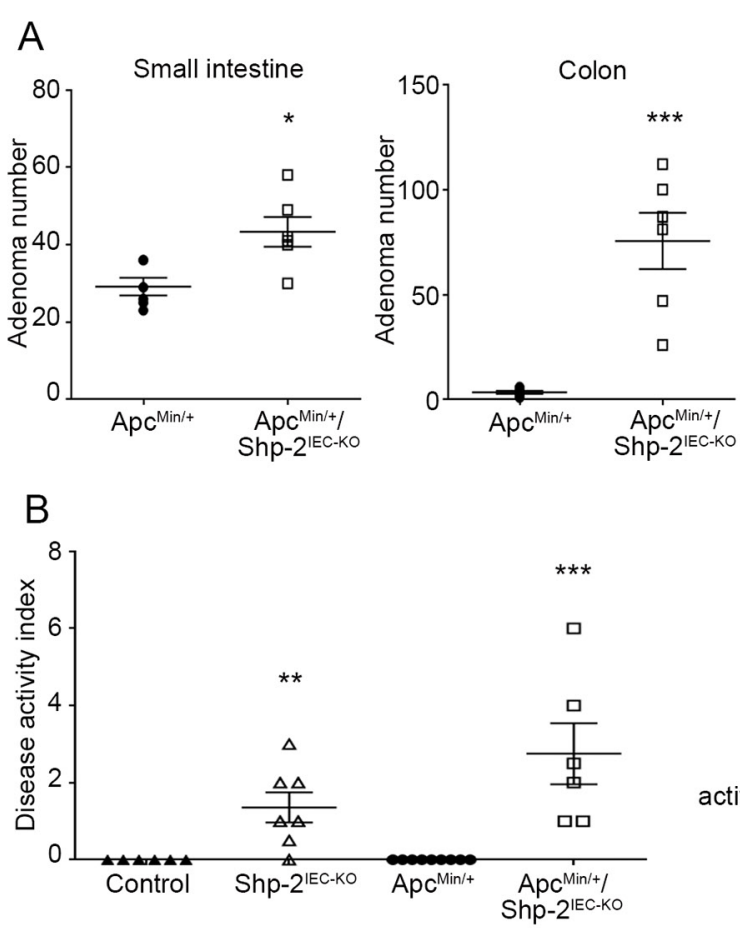

C
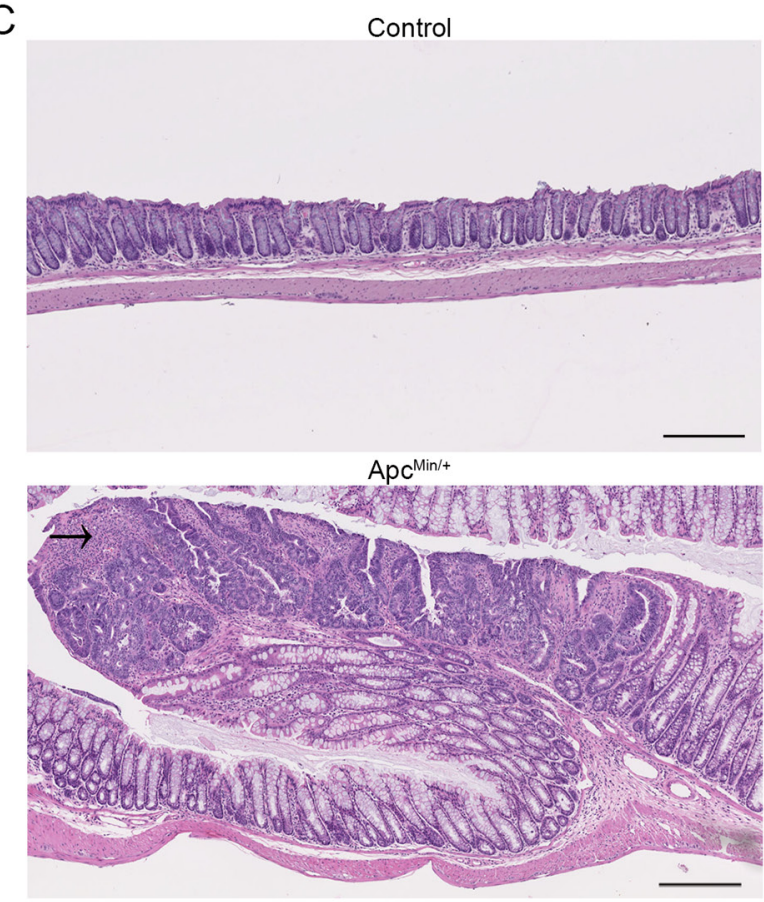

$\mathrm{D}$
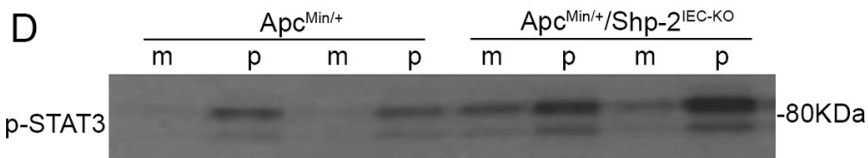

STAT3

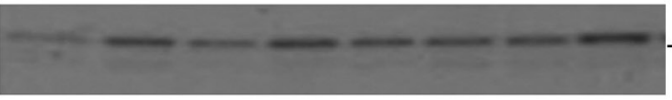

$-80 \mathrm{KDa}$
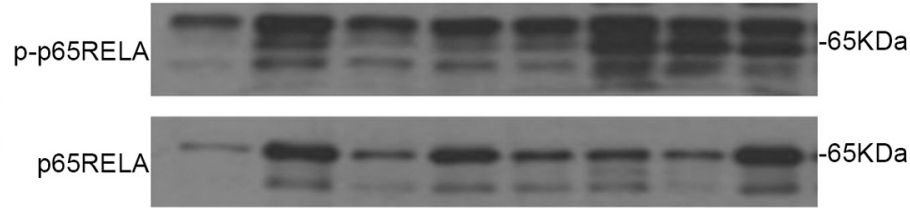

$65 \mathrm{KDa}$

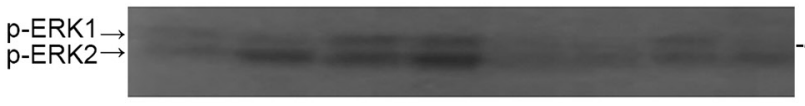

$44 \mathrm{KDa}$

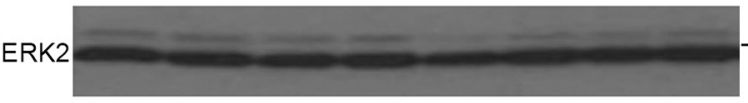

$44 \mathrm{KDa}$
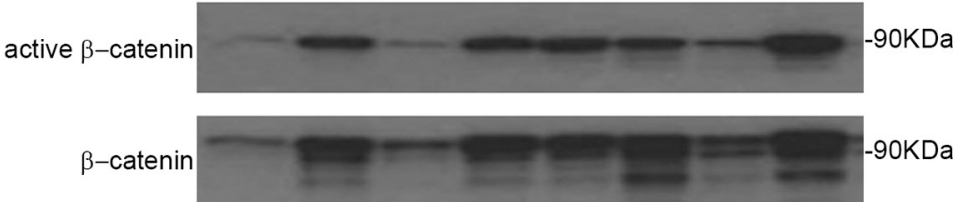

-90KDa
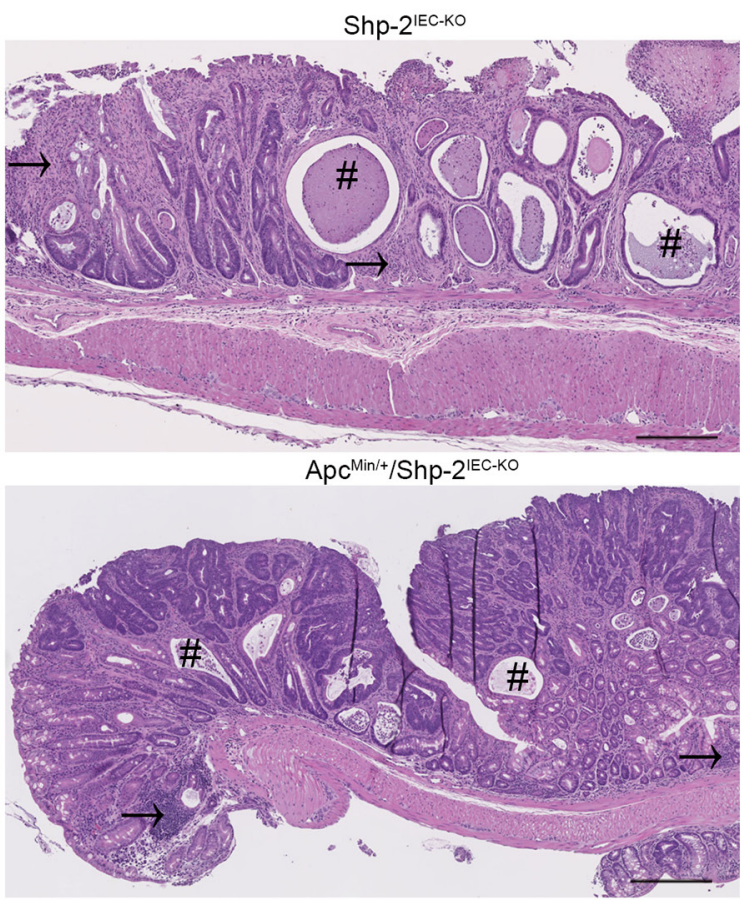

Figure 9: IEC-specific Shp-2 ablation exacerbates tumorigenesis in $\boldsymbol{A p} \boldsymbol{c}^{\mathrm{Min} / \mathbf{+}}$ mice. A. The number of polyps formed in the small intestine and colon was determined at 3 months of age in both $A p c^{\operatorname{Min} /+}$ and $A p c^{\operatorname{Min} /+}$ mice lacking Shp-2 expression $\left(A p c^{M i n /+} ; S_{h p} 2^{I E C-K O}\right.$ mice) $(\mathrm{N}=6)$. B. Disease activity index of 3 month-old mice was calculated by scoring stool softness, occult fecal blood, rectal bleeding and colon rigidity $(\mathrm{N}=6)$. C. Representative images of 3 month-old control Shp-2 IEC-KO,$A p c^{M i n /+}$ and $A p c^{M i n /+}$; Shp- $2^{I E C-K O}$ murine colons. \# indicates crypt abscesses and arrow shows immune cell infiltration. Scale bars: $100 \mu \mathrm{m}$. D. Western blot analyses were performed on paired specimens of margins (m) and polyps (p) extracted from $A p c^{M i n /+}$ and $A p c^{M i n /+}$; Shp- $2^{I E C-K O}$ mice. Levels of total and phosphorylated ERK1/2 (T202/Y204), STAT3 (Y705) and p65NFkB (S536) as well as total and non-phospho (active) $\beta$-catenin were specifically analyzed. Representative immunoblot are shown. Data are expressed as mean $\pm \mathrm{SEM}$. ${ }^{*} \mathrm{p} \leq 0.05,{ }^{*} \mathrm{p} \leq 0.01,{ }^{* * *} \mathrm{p} \leq 0.001$. 
even in the presence of oncogenic RAS [47]. Recent results have further demonstrated that SHP-2 binds and dephosphorylates both wild-type and oncogenic HRAS on Tyr32 in astrocytes and glioma cells, an event that increases RAS association with RAF-1 and downstream MEK/ERK activation [48]. Herein, dysregulated phosphorylation of RAF-1 was observed in SHP-2 deficient cells expressing oncogenic KRAS. Since Tyr32 is conserved in human and mouse KRAS proteins, our data suggest that SHP-2 may also target KRAS in IECs, enhancing the stimulation of downstream proliferative RAS/MEK/ERK kinases.

The somatic E76G mutation specifically found in colorectal cancer specimens disrupts the inhibitory intramolecular interaction within the PTP domain and leads to the hyperactivation of SHP-2 [40]. Given the latter, we speculated that by inducing the RAS/ERK signaling pathway, expression of the SHP-2 E76 mutant in IECs would be sufficient to trigger intestinal neoplasia. However, Ptpn 11 E76K knock-in mice failed to develop any intestinal tumors after 1 year. These findings hence indicate that SHP-2 does not function as a classical oncogene in intestinal epithelium. SHP-2 may need to cooperate with additional oncogenic events such as the loss of the $A p c$ gene to initiate intestinal neoplasia. Our findings hence support the model in which constitutive activation of SHP-2 drives the development of adenoma in the intestine of $A p c^{\mathrm{Min} /+}$ mice. SHP-2 expression appears to be required for full activation of ERK $1 / 2$ both in normal intestinal epithelium [14] and Apc-mutated epithelium (Figure 9D). An important role of the ERK pathway in the establishment of the Min phenotype has moreover been previously described [31]. Therefore, in addition to the inactivation of $A p c$, activation of ERK signaling, either by the activation of SHP-2 as demonstrated herein or by mutation of KRAS [49], appears to act synergistically resulting in increased tumor multiplicity in the intestine.

In addition, we further demonstrate that mice with conditional deletion of Shp-2 in IECs developed colorectal adenocarcinomas with age. These adenocarcinomas exhibited several features that distinguish colitisassociated cancer including mucosal ulcerations, regenerative proliferation and persistent histological inflammation [50]. Likewise, signs of oxidative stress (increased Nox1, Ho-1, iNos and Cox-2) and DNA damage (phospho-H2AX) were also observed in the colon of aged Shp-2 $2^{I E C-K O}$ mice. A major mechanism that links inflammation to proneoplastic genetic alterations involved in the pathogenesis of CAC is oxidative stress [50]. Oxidative stress is mainly produced by cells of the innate immune system such as macrophages and granulocytes which generate various reactive oxygen and nitrogen species (ROS, NOS) which impose a persistent mutational pressure to IECs, resulting in DNA breaks and adducts. In this regard, base transversions in TP53 gene were often observed in UC patients who develop CAC, and oxidative
DNA damage may presumably be responsible for these errors [36,51]. Accordingly, increased levels of the mutant form of p53 $(\mathrm{R} 273 \mathrm{H})$, a hot spot residue in CRC [52], were detected in inflamed mucosae from aged Shp-2IEC-KO mice. Moreover, in recent years, major pro-inflammatory pathways have been implicated in inflammation-associated tumor development. Among these, the transcription factors NFKB and STAT3 have taken center stage [1617]. Indeed, Greten et al. showed that deletion of $I K K \beta$ in IECs resulted in decreased numbers of tumors and increased apoptosis in the azoxymethane (AOM)-dextran sodium sulfate (DSS) model [53]. Besides, specific STAT3 ablation in IECs interfered with tumor formation and tumor growth in the AOM-DSS model [33]. Conversely, IEC-specific Socs 3 ablation or expression of the SOCS3binding deficient $g p 130^{Y 757 F}$ mutation both inducing STAT3 activation, promoted colonic tumor growth and incidence [33, 54]. Thus, tumor cell-specific NFkB and STAT3 signalings appear to be required for inflammationassociated tumor initiation in the colon [2], likely by inducing the expression of pro-proliferative, anti-apoptotic and immune response genes [33]. Furthermore, activated $\mathrm{NF} \kappa \mathrm{B}$ can suppress p53 acetylation and transactivation through sequestration of CBP or p300 [55], inhibiting p53 tumor suppressor function [56]. Interestingly, $\mathrm{NF} \kappa \mathrm{B}$ and STAT3 have both been shown to be hyperphosphorylated in the colonic epithelium of 1-month [14] and 15-monthold Shp-2 $2^{I E C-K O}$ mice. Furthermore, levels of acetylated p53 were markedly downregulated in the colon of these mice, thereby creating permissive conditions for oncogenic transformation. The exact mechanism by which Shp-2 ablation in IECs induces NFאB and STAT3 activation is not totally clear. However, we can speculate that this is an IEC-intrinsic consequence of $S h p-2$ inactivation since SHP-2 silencing in cultured IECs increases the levels of phosphorylated STAT3 and p65RELA [14]. Furthermore, SHP-2 can closely regulate phosphorylation and activation of both of these transcription factors in several cell types [9].

Paradoxically, activation of NFאB and STAT3 in IECs specifically has been demonstrated to protect the gut against inflammation. Indeed, conditional IEC-specific deletion of NEMO (essential for NFאB activation) spontaneously caused colon inflammation [57]. Likewise, mice with deletion of STAT3 in IECs showed increased susceptibility to DSS [58] and to Citrobacter rodentium infection [59]. Hence, both NFאB and STAT3 expressed in IECs exert a protective, homeostatic function under physiological conditions [57]. Therefore, this indicates that Shp-2 $2^{I E C-K O}$ mice develop colitis despite the rapid and persistent activation of STAT3 and NFאB in IECs. We cannot exclude however that excessive activation of STAT3 and/or NFאB might also contribute to the pathogenesis of the inflammatory phenotype in Shp$2^{I E C-K O}$ mice [60-61]. Nevertheless, by inducing chronic inflammation as well as STAT3 and NFאB activation, 
IEC-specific deletion of Shp-2 results in the formation and growth of pre-malignant and malignant lesions typical of colitis-associated carcinogenesis.

Our data also reveal that SHP-2 inactivation in IECs considerably exacerbated mutant $A p c$-initiated transformation, notably shifting tumor incidence toward the colon. Similarly, 1-week treatment of $A p c^{\mathrm{Min} /+}$ mice with Dextran Sulfate Sodium (DSS), which causes acute colonic inflammation, was found to markedly increase the multiplicity and incidence of colonic neoplasms in these mice [62]. These findings thus reinforce the notion that SHP-2 exerts a tumor suppressive action in the colon by inhibiting the development of inflammation [14], probably through the maintenance of appropriate colonic barrier function [63]. Such anti-oncogenic action of SHP-2 was also recently described in a mouse model of liver cancer [15] in which mice with hepatocyte-specific deletion of Shp-2 developed severe inflammation in the liver leading to tumor development.

Our study thus demonstrated that both activation and inactivation of Shp-2 in mouse colonic epithelium promotes tumor development. Such dual role for Shp-2 in cancer development was also previously proposed in the liver [64-65]. However, it is important to emphasize that inactivation of Shp-2 in the colon epithelium was much more detrimental for the mice than the activation of Shp-2. Indeed, Shp- $2^{I E C-K O}$ mice showed severe diarrhea and rectal bleeding with higher mortality in comparison to controls. By contrast, no difference on survival rate was noticed between $S h p-2^{I E C-E 76 K}$ mice and their control littermates. Furthermore, all $S h p-2^{I E C-K O}$ mice developed colorectal adenocarcinomas with age; however, no $S h p-2^{I E C-E 76 K}$ have developed intestinal neoplasia after 15 months of age. Lastly, colonic tumor multiplicity in $\mathrm{Apc}^{\mathrm{Min} /+}$ mice was much more increased by $S h p-2$ deletion (35-fold) than by Shp-2 activation (3-fold).

Although the molecular mechanisms underlying the opposing actions of SHP-2 in IECs remain to be clarified, they may predominantly rely on the ability of SHP-2 to activate the ERK signaling. Indeed, the ERK pathway, which is consistently stimulated upon activation of SHP-2 $[8-10,14]$, is commonly associated with intestinal crypt cell proliferation and tumorigenesis $[31,66]$. Conversely, activation of ERK in secretory progenitors has been recently shown to direct Goblet cell differentiation $[63,67]$ and these cells are crucial for the maintenance of barrier function and the prevention of intestinal inflammation [68]. More specifically, Heuberger et al. also found that ERK signaling controls secretory cell fate in the small intestine by negatively regulating $\mathrm{Wnt} / \beta$-catenin activity. Unfortunately, they did not analyze $\beta$-catenin protein expression but they showed, in intestinal organoids and cultured cells, that ERK inhibition changed the relative abundance of Tcf4 isoforms [67]. How exactly SHP2 regulates $\beta$-catenin protein levels remains therefore to be examined. Intriguingly, we previously reported that ectopic expression of SHP-1, a SHP-2 paralog, stimulates $\beta$-catenin proteasomal degradation, decreasing its transcriptional activity [69]. Current experiments are in progress to verify if SHP-2 can similarly regulate $\beta$-catenin stability and activity in cultured IECs.

In conclusion, the present study reveals that SHP2 exerts opposing functions, pro-oncogenic versus antioncogenic (by inhibiting inflammation), in the colon epithelium. These opposing functions for SHP-2 may depend on cellular context (transit-amplifying cells, secretory progenitor cells). Further studies are required to identify the binding partner proteins of SHP-2 that may direct these opposing cellular responses under physiological and pathological conditions.

\section{MATERIAL AND METHODS}

\section{Materials}

Antibodies. Primary antibodies were obtained from the following sources: SHP-2, MEK1/2, ERK2, phosphoRAF1 (Y340/Y341), RAF1, KI67 and $\gamma$ H2AX from Santa Cruz Biotechnology (Santa Cruz, CA, USA), phosphoERK1/2 (T202/Y204) from Sigma-Aldrich (Mississauga, ON, Canada), $\beta$-actin from Chemicon International (Billerica, MA, USA), GAPDH (HRP conjugate), phospho-RELA (S536), RELA, phospho-STAT3 (Y705), STAT3, phospho-RAF-1 (S259), phospho-RAF-1 (S338), non-phospho (active) $\beta$-catenin, phospho-AKT (S473), AKT, acetylated-p53 (K379) and phospho-MEK1/2 (S218/S222) from Cell Signaling Technology (Danvers, MA, USA), total p53 and mutant-p53 (R273H) from Abcam (Toronto, ON, Canada) and $\beta$-catenin from BD Pharmingen (Mississauga, ON, Canada). Mouse and rabbit horseradish peroxidase antibodies were purchased from Amersham Biosciences (Pittsburg, PA, USA). All other materials were from Sigma-Aldrich (Oakville, ON, Canada) unless stated otherwise.

\section{Human colorectal tissues}

Ninety four specimens of colon tumors and paired normal tissues (at least $10 \mathrm{~cm}$ from the tumor) were obtained from patients undergoing surgical resection and were processed as previously described [70]. Patients did not receive neoadjuvant therapy. Tissues were collected after obtaining the patient's written informed consent, according to the protocol approved by the Institutional Human Subject Review Board of the Centre Hospitalier Universitaire de Sherbrooke. Pathological and clinical data were obtained from medical records and are provided in Supplementary Table S1.

\section{Cell culture}

The rat intestinal epithelial crypt cells IEC-6 stably overexpressing empty vector (pBabepuro) or $\mathrm{KRAS}^{\mathrm{G} 12 \mathrm{~V}}$ mutant were generated after retroviral infection and were 
previously characterized and cultured as described [23]. The colon carcinoma cell lines SW480, HT29 and DLD-1 were cultured as described [70-71]. HT-29 and SW480 cell lines were purchased from ATCC (Manassas, VA, USA) in October 2014 and DLD-1 were authenticated using Cell authentication service from ATCC in October 2014.

\section{Generation of shRNA, production of lentiviruses production and cell infection or transfection}

The lentiviral shRNA expression vector (pLenti6-U6) was constructed as described [60]. Sequences of rat shRNA oligonucleotides are available upon request [14]. Irrelevant pLenti-shRNA with the scrambled rat shShp-2 sequence was used as negative control (shControl). Lentiviruses produced in 293T cells were used for infection of IEC-6 cells according to Invitrogen recommendations. To knock down SHP-2 in human colorectal cancer cells, cells were transfected using Lipofectamine 2000 (Invitrogen, Waltham, MA, USA) with 50nMol SHP-2 siRNA corresponding to fw5'UAAAUCGGUACUGUGCUUCUGUCUG-3' and rev5'CAGACAGAAGCACAGACCGAUUUA-3' for siRNA\#1 or to fwd5'-AAUAUUUGUAUAUUCGUGCCCUUUC3' and rev5'GAAAGGGCACGAAUAUACAAAUAUU-3' for siRNA\#2 (IDT, Coralville, IA, USA) as described by Wang HC et al. [72]. A scrambled siRNA was also used as a control (siCTL-Invitrogen Stealth ${ }^{\mathrm{TM}}$ RNAi Negative Control LOGC).

\section{Western blot analysis}

Protein extractions and Western blot analyses were performed as described [14, 24].

\section{Cell proliferation, soft agarose and migration/ invasion assays}

Experiments were started 7 days post-selection (for pLenti-shRNA infection) or 48 hours post transfection (for RNAi). For growth assays, cells were seeded in 6 -well plates $\left(3.5 \times 10^{4}\right.$ cells/well $)$. The number of cells was calculated daily during 7 days using a Cell particle counter (Invitrogen). Soft agarose and migration/invasion assays (Biocoat Matrigel, Becton Dickinson, Bedford, MA, USA) were performed as reported [70]. Cells were visualized by inverted light microscopy (Zeiss apparatus) and crystal violet stained inserts were visualized by light microscopy (Leica apparatus). Cell count was obtained with ImageJ software. For all experiments, three different cell populations originating from three different infections (shRNA) or transfections (siRNA) were analyzed.

\section{Immunocompromised mice}

CD1 nu/nu mice were purchased from Charles River Laboratory (Wilmington, MA, USA). Mice were housed in individually-ventilated cages. Food, water, bedding and cages were sterilized. Experiments were approved by the Animal Research Committee of the Faculty of Medicine and Health Sciences of the Université de Sherbrooke. A total of $5 \times 10^{5}$ cells suspended in $0.1 \mathrm{ml}$ DMEM were injected into the dorsal subcutaneous tissue of 7-week-old female mice. Tumor volume was determined every 3 days by external measurement according to the formula $\left(d^{2} \times D\right) / 2$. Three different cell populations originating from three different viral infections and different virus productions were assessed in minimally four different mice per population, for a total of 13 mice.

\section{Conditional KO and KI mice}

Shp-2 floxed allele mice [14] were previously crossed with Villin-Cre mice (Jackson Laboratory) to specifically delete $S h p-2$ in the intestinal epithelium (referred as Shp-2 ${ }^{I E C-K O}$ ) [14]. Knock-in Ptpn11 ${ }^{E 76 K /+}$ mice [29] (on C57BL6/J background) were crossed with Villin-Cre mice to specifically express heterozygote Shp $-2^{E 76 K /+}$ mutant in the intestinal epithelium (referred as $\left.S h p-2^{I E C-E 76 K}\right)$. Only homozygote $S h p-2^{+/+}$mice were used as control littermates. $\mathrm{C} 57 \mathrm{BL} 6 / \mathrm{J}-A p c^{\mathrm{Min} /+}$ mice were obtained from Jackson Laboratory (Bar Harbor, ME, USA). These mice were mated to generate $A p c^{\mathrm{Min} /+}$; Shp-2 $2^{I E C-K O}$ and $A p c^{M i n /+} ; S h p-2^{I E C-E 76 K}$ models. The first cross was between the Villin-Cre and $A p c^{\mathrm{Min} /+}$ mice. The double heterozygous mice were then bred with Shp $2^{\text {flox/flox }}$ mice one time and the triple heterozygous mice were finally bred with $\operatorname{Sh} p 2^{\text {flox/flox }}$ mice to generate the experimental mice $\left(A p c^{\mathrm{Min} /+} ; S h p-2^{I E C-K O}\right)$ and control littermates. The double heterozygous $\mathrm{Apc}^{\mathrm{Min} /+}$; Villin-Cre mice were also bred with $P t p n 11^{E 76 K /+}$ mice to generate the triple heterozygous experimental mice and their control littermates. Genomic DNA was isolated using the Spin Doctor genomic DNA kit from Gerard Biotech following the manufacturer's protocol. For the knock-in Shp-2 $2^{I E C-E 76 K}$ mice, loss of Neo cassette was measured by PCR analysis to validate the $S h p-2^{\mathrm{E} 76 \mathrm{~K}}$ expression in intestinal tissue [29]. PCR conditions and primer sequences used for genotyping are available upon request. All experiments in mice were approved by the Animal Research Ethics Committee of the Faculty of Medicine and Health Sciences of the Université de Sherbrooke.

\section{Histological staining, immunohistochemistry and macroadenoma counts}

Colons were fixed, sectioned and stained as described previously [73]. Fibrosis was visualized using Trichrome Stain (Masson) Kit (Sigma-Aldrich). Polyps were counted and measured in the gastrointestinal tract from stomach to rectum of control and mutant $A p c^{\mathrm{Min} /+}$ littermates as previously described [73-74]. 


\section{RNA extraction and quantitative RT-PCR analysis}

RNA was isolated from the scraped colonic mucosa of mice using the RNeasy minikit (Qiagen). RT-PCR was performed using AMV-RT (Roche Diagnostics) according to the manufacturer's instructions and quantitative PCR was performed by the RNomics Platform at the Université de Sherbrooke (Sherbrooke, QC, Canada). All primer sequences and cycling conditions are available upon request.

\section{Statistical analysis and figure editing}

All assays were minimally performed in triplicate. Typical results shown are representative of 3 independent experiments and data are expressed as mean \pm SEM. Densitometric analysis was performed using ImageJ software. Results were analyzed using the Student's $t$-test and were considered statistically significant at $p \leq 0.05$. Graphs and statistics were generated with Graphpad Prism (Graphpad Software Inc., LaJolla, CA) and figures were constructed using Photoshop software.

\section{Abbreviations}

AOM, azoxymethane; APC, adenomatous polyposis coli; CAC, colitis-associated cancer; Cox-2, cyclooxygenase-2; CRC, colorectal cancer; DSS, dextran sulfate sodium; EGF, epidermal growth factor; ERK, extracellular signal regulated kinase; IBD, inflammatory bowel diseases; IEC, intestinal epithelial cell; IKK $\beta$, IкB kinase; iNOS, JAK, Janus-associated kinase; JNK, c-jun N-terminal kinase; MAPK, mitogen-activated protein kinase; NFkB, nuclear factor kappa B; NOS, nitrogen oxygen species; NSCLC, non-small cell lung cancer; PI3K, phosphatidylinositol 3-kinase; RELA, v-rel reticuloendotheliosis viral oncogene homolog A; ROS, reactive oxygen species; Ser, serine; SHP-2, Src homology 2-domain-containing phosphatase; shRNA: short hairpin RNA; SOCS3, suppressor of cytokine signaling 3; STAT, signal transducer and activator of transcription; Tyr, tyrosine; UC, Ulcerative colitis;

\section{ACKNOWLEDGMENTS}

We thank Claire Hamel and Gérald Bernatchez for technical assistance. We thank the Electron Microscopy \& Histology Research Core of the FMSS at the Université de Sherbrooke for histology, electron microscopy, and phenotyping services.

\section{CONFLICTS OF INTEREST}

The authors declare that they have no competing interests.

\section{GRANT SUPPORT}

The biobank of colorectal cancer specimens was supported by a Team grant on digestive epithelium from the Canadian Institutes of Health Research. Jessica Gagné-Sansfaçon is student scholar from the Fonds de la Recherche en Santé du Québec (FRSQ). Nathalie Rivard and Julie C Carrier are members of the FRSQFunded Centre de Recherche du CHUS. Nathalie Rivard is a recipient of a Canadian Research Chair in colorectal cancer and inflammatory cell signaling.

This research was supported by grants from the Cancer Research Society and Canadian Institutes of Health Research to NR

\section{REFERENCES}

1. Grivennikov SI. Inflammation and colorectal cancer: colitisassociated neoplasia. Semin Immunopathol. 2013; 35: 229-244.

2. Foersch S, Waldner MJ and Neurath MF. Colitis and colorectal cancer. Dig Dis. 2012; 30: 469-476.

3. Smith G, Carey FA, Beattie J, Wilkie MJ, Lightfoot TJ, Coxhead J, Garner RC, Steele RJ and Wolf CR. Mutations in APC, Kirsten-ras and p53--alternative genetic pathways to colorectal cancer. Proc Natl Acad Sci USA. 2002; 99: 9433-9438.

4. George B and Kopetz S. Predictive and prognostic markers in colorectal cancer. Curr Oncol Rep. 2011; 13: 206-215.

5. Thorsteinsdottir S, Gudjonsson T, Nielsen Ole H, Vainer $\mathrm{B}$ and Seidelin JB. Pathogenesis and biomarkers of carcinogenesis in ulcerative colitis. Nat Rev Gastroenterol Hepatol. 2011; 8: 395-404.

6. Narumi $\mathrm{Y}$, Isomoto $\mathrm{H}$, Shiota $\mathrm{M}$, Sato $\mathrm{K}$, Kondo $\mathrm{S}$, Machida H, Yanagihara K, Mizuta Y, Kohno S and Tsukamoto K. Polymorphisms of PTPN11 coding SHP-2 as biomarkers for ulcerative colitis susceptibility in the Japanese population. J Clin Immunol. 2009; 29: $303-310$

7. Grossmann KS, Rosário M, Birchmeier $\mathrm{C}$ and Birchmeier W. The tyrosine phosphatase Shp2 in development and cancer, Adv Cancer Res. 2010; 106: 53-89

8. Qu CK, Yu WM, Azzarelli B and Feng GS. Genetic evidence that Shp-2 tyrosine phosphatase is a signal enhancer of the epidermal growth factor receptor in mammals. Proc Natl Acad Sci USA. 1999; 96: 8528-8533.

9. Neel BG, Gu H and Pao L. The 'Shp'ing news: SH2 domaincontaining tyrosine phosphatases in cell signaling. Trends Biochem Sci. 2003; 28: 284-293.

10. Rivard N, McKenzie FR, Brondello JM and Pouysségur J. The phosphotyrosine phosphatase PTP1D, but not PTP1C, is an essential mediator of fibroblast proliferation induced 
by tyrosine kinase and G protein-coupled receptors. J Biol Chem. 1995; 270: 11017-11024.

11. Xu D and Qu CK. Protein tyrosine phosphatases in the JAK/STAT pathway. Front Biosci. 2008; 13: 4925-4932.

12. Ivins ZC, Kontaridis MI, Fornaro M, Feng GS and Bennett AM. SHP-2 regulates the phosphatidylinositide 3'-kinase/ Akt pathway and suppresses caspase 3-mediated apoptosis. J Cell Physiol. 2004; 199: 227-236.

13. Kontaridis MI, Eminaga S, Fornaro M, Zito CI, Sordella R, Settleman J and Bennett AM. SHP-2 positively regulates myogenesis by coupling to the Rho GTPase signaling pathway. Mol Cell Biol. 2004; 24: 5340-5352.

14. Coulombe G, Leblanc C, Cagnol S, Maloum F, Lemieux E, Perreault N, Feng GS, Boudreau F and Rivard N. Epithelial tyrosine phosphatase SHP-2 protects against intestinal inflammation in mice. Mol Cell Biol. 2013; 33: 2275-2284.

15. Bard-Chapeau EA, Li S, Ding J, Zhang SS, Zhu HH, Princen F, Fang DD, Han T, Bailly-Maitre B, Poli V, Varki NM, Wang H and Feng GS. Ptpn11/Shp2 acts as a tumor suppressor in hepatocellular carcinogenesis. Cancer Cell. 2011; 19: 629-639.

16. Waldner MJ and Neurath MF. Mechanisms of immune signaling in colitis-associated cancer. Cell Mol Gastroenterol Hepatol. 2014; 1: 6-16.

17. Viennois E, Chen F and Merlin D. NF-kB pathway in colitis-associated cancers. Transl Gastrointest Cancer. 2013; 1: 21-29.

18. Bentires-Alj M, Paez JG, David FS, Keilhack H, Halmos B, Naoki K, Maris JM, Richardson A, Bardelli A, Sugarbaker DJ, Richards WG, Du J, Girard L et al. Activating mutations of the Noonan syndrome-associated SHP2/PTPN11 gene in human solid tumors and adult acute myelogenous leukemia. Cancer Res. 2004; 64: 8816-8820.

19. Miyamoto D, Miyamoto M, Takahashi A, Yomogita Y, Higashi H, Kondo S and Hatakeyama M. Isolation of a distinct class of gain-of-function SHP-2 mutants with oncogenic RAS-like transforming activity from solid tumors. Oncogene. 2008; 27: 3508-3515.

20. Zheng H, Li S, Hsu P and Qu CK. Induction of a tumorassociated activating mutation in protein tyrosine phosphatase Ptpn11 (Shp2) enhances mitochondrial metabolism, leading to oxidative stress and senescence. J Biol Chem. 2013; 288: 25727-25738.

21. Malumbres M and Barbacid M. RAS oncogenes: the first 30 years. Nat Rev Cancer. 2003; 3: 459-465.

22. Pretlow TP and Pretlow TG. Mutant KRAS in aberrant crypt foci (ACF): initiation of colorectal cancer? Biochim Biophys Acta. 2005; 1756: 83-96.

23. Cagnol S and Rivard N. Oncogenic KRAS and BRAF activation of the MEK/ERK signaling pathway promotes expression of dual-specificity phosphatase 4 (DUSP4/ MKP2) resulting in nuclear ERK1/2 inhibition. Oncogene. 2013; 32: 564-576.
24. Lemieux E, Cagnol S, Beaudry K, Carrier J and Rivard N. Oncogenic KRAS signalling promotes the Wnt/ $\beta$-catenin pathway through LRP6 in colorectal cancer. Oncogene. 2015; 34: 4914-4927.

25. Ikenoue T, Kanai F, Hikiba Y, Obata T, Tanaka Y, Imamura J, Ohta M, Jazag A, Guleng B, Tateishi K, Asaoka Y, Matsumura M, Kawabe $\mathrm{T}$ et al. Functional analysis of PIK3CA gene mutations in human colorectal cancer. Cancer Res. 2005; 65: 4562-4567.

26. Li WQ, Kawakami K, Ruszkiewicz A, Bennett G, Moore $\mathrm{J}$ and Lacopetta B. BRAF mutations are associated with distinctive clinical, pathological and molecular features of colorectal cancer independently of microsatellite instability status. Mol Cancer. 2006; 5: 2.

27. Lavoie $H$ and Therrien M. Regulation of RAF protein kinases in ERK signalling. Nat Rev Mol Cell Biol. 2015; 16: 281-298.

28. Jelinek T, Dent P Sturgill TW and Weber MJ. Rasinduced activation of Raf-1 is dependent on tyrosine phosphorylation. Moll Cell Biol. 1996; 3: 1027-1034.

29. Xu D, Liu X, Yu WM, Meyerson HJ, Guo C, Gerson SL and Qu CK. Non-lineage/stage-restricted effects of a gainof-function mutation in tyrosine phosphatase Ptpn11 (Shp2) on malignant transformation of hematopoietic cells. J Exp Med. 2011; 208: 1977-1988.

30. Moser AR, Pitot HC and Dove WF. A dominant mutation that predisposes to multiple intestinal neoplasia in the mouse. Science. 1990; 247: 322-324.

31. Lee SH, Hu LL, Gonzalez-Navajas J, Seo GS, Shen C, Brick J, Herdman S, Varki N, Corr M, Lee J and Raz E. ERK activation drives intestinal tumorigenesis in Apc(Min/+) mice. Nat Med. 2010; 16: 665-670.

32. Fantini $\mathrm{MC}$ and Pallone F. Cytokines: from gut inflammation to colorectal cancer. Curr Drug Targets. 2008; 5: 375-380.

33. Grivennikov S, Karin E, Terzic J, Mucida D, Yu GY, Vallabhapurapu S, Scheller J, Rose-John S, Cheroutre H, Eckmann L and Karin M. IL-6 and Stat3 are required for survival of intestinal epithelial cells and development of colitis-associated cancer. Cancer Cell. 2009; 15: 103-113.

34. Atreya R and Neurath MF. Involvement of IL-6 in the pathogenesis of inflammatory bowel disease and colon cancer. Clin Rev Allergy Immunol. 2005; 3: 187-196.

35. Francescone R, Hou V and Grivennikov SI. Cytokines, IBD, and colitis-associated cancer. Inflamm Bowel Dis. 2015; 21: 409-418.

36. Seril DN, Liao J, Yang GY and Yang CS. Oxidative stress and ulcerative colitis-associated carcinogenesis: studies in humans and animal models. Carcinogenesis. 2003; 24: 353-362.

37. Hussain SP, Amstad P, Raja K, Ambs S, Nagashima M, Bennett WP, Shields PG, Ham AJ, Swenberg JA, Marrogi $\mathrm{AJ}$ and Harris CC. Increased p53 mutation load in 
noncancerous colon tissue from ulcerative colitis: a cancerprone chronic inflammatory disease. Cancer Res. 2000; 60: 3333-3337.

38. Noguchi T, Matozaki T, Horita K, Fujioka Y and Kasuga M. Role of SH-PTP2, a protein-tyrosine phosphatase with Src homology 2 domains, in insulin-stimulated Ras activation. Mol Cell Biol. 1994; 14: 6674-6682.

39. Shi ZQ, Yu DH, Park M, Marshall M and Feng GS. Molecular mechanism for the Shp-2 tyrosine phosphatase function in promoting growth factor stimulation of Erk activity. Mol Cell Biol. 2000; 20:1526-1536.

40. Zheng H, Alter S and Qu CK. SHP-2 tyrosine phosphatase in human diseases. Int J Clin Exp Med. 2009; 2: 17-25.

41. Coulombe G and Rivard N. New and unexpected biological functions for the Src-Homology 2 Domain-Containing Phosphatase SHP-2 in the gastrointestinal tract. Cell Mol Gastroenterol Hepatol. 2015; 2: 11-21.

42. Huang WQ, Lin Q, Zhuang X, Cai LL, Ruan RS, Lu ZX and Tzeng CM. Structure, function, and pathogenesis of SHP2 in developmental disorders and tumorigenesis. Curr Cancer Drug Targets. 2014; 14: 567-588.

43. Cai P, Guo W, Yuan H, Li Q, Wang W, Sun Y, Li X and $\mathrm{Gu}$ Y. Expression and clinical significance of tyrosine phosphatase SHP-2 in colon cancer. Biomed Pharmacother. 2014; 68: 285-290.

44. Villanueva DA, Mallona I and Peinado MA. Wanderer, an interactive viewer to explore DNA methylation and gene expression data in human cancer. Epigenetics Chromatin. $2015 ; 8: 22$.

45. Smakman N, Veenendaal LM, van Diest P, Bos R, Offringa R, Borel Rinkes IH and Kranenburg O. Dual effect of Kras (D12) knockdown on tumorigenesis: increased immunemediated tumor clearance and abrogation of tumor malignancy. Oncogene. 2005; 24: 8338-8342.

46. Langlois MJ, Bergeron S, Bernatchez G, Boudreau F, Saucier C, Perreault N, Carrier JC and Rivard N. The PTEN phosphatase controls intestinal epithelial cell polarity and barrier function: role in colorectal cancer progression. PLoS One. 2010; 5:e15742

47. Yamauchi K, Milarski KL, Saltiel AR and Pessin JE. Protein-tyrosine-phosphatase SHPTP2 is a required positive effector for insulin downstream signaling. Proc Natl Acad Sci USA. 1995; 92: 664-668.

48. Bunda S, Burrell K, Heir P, Zeng L, Alamsahebpour A, Kano Y, Raught B, Zhang ZY, Zadeh G and Ohh M. Inhibition of SHP2-mediated dephosphorylation of Ras suppresses oncogenesis. Nat Commun. 2015; 6: 8859.

49. Janssen KP, Alberici P, Fsihi H, Gaspar C, Breukel C, Franken P, Rosty C, Abal M, El Marjou F, Smits R, Louvard D, Fodde R and Robine S. APC and oncogenic KRAS are synergistic in enhancing Wnt signaling in intestinal tumor formation and progressional. Gastroenterology. 2006; 131: 1096-1109.
50. Rogler G. Chronic ulcerative colitis and colorectal cancer. Cancer Lett. 2014; 345: 235-241.

51. Martinez CA, Bartocci PC, do Carmo CV, Pereira JA, Miranda DD, Ribeiro ML. The effects of oxidative DNA damage and mutations in the $\mathrm{p} 53$ protein on cells of the colonic mucosa with and without the fecal stream: an experimental study in rats. Scand J Gastroenterol. 2010; 45:714-24.

52. Liu Y and Bodmer FW. Analysis of P53 mutations and their expression in 56 colorectal cancer cell lines. Proc Natl Acad Sci U S A. 2006; 103:976-81.

53. Greten FR, Eckmann L, Greten TF, Park JM, Li ZW, Egan LJ, Kagnoff MF and Karin M. IKKbeta links inflammation and tumorigenesis in a mouse model of colitis-associated cancer. Cell. 2004; 3: 285-296.

54. Bollrath J, Phesse TJ, von Burstin VA, Putoczki T, Bennecke M, Bateman T, Nebelsiek T, Lundgren-May T, Canli O, Schwitalla S, Matthews V, Schmid RM, Kirchner $\mathrm{T}$ et al. gp130-mediated Stat3 activation in enterocytes regulates cell survival and cell-cycle progression during colitis-associated tumorigenesis. Cancer Cell. 2009; 15: 91-102.

55. Webster GA and Perkins ND. Transcriptional cross talk between NF-kappaB and p53. Mol Cell Biol. 1999; 19: 3485-3495.

56. Gudkov AV, Gurova KV and Komarova EA. Inflammation and p53: A Tale of Two Stresses. Genes Cancer. 2011; 2: 503-516. doi: 10.1177/1947601911409747.

57. Nenci A, Becker C, Wullaert A, Gareus R, van Loo G, Danese S, Huth M, Nikolaev A, Neufert C, Madison B, Gumucio D, Neurath MF and Pasparakis M. Epithelial NEMO links innate immunity to chronic intestinal inflammation. Nature. 2007; 446:557-61.

58. Pickert G, Neufert C, Leppkes M, Zheng Y, Wittkopf N, Warntjen M, Lehr HA, Hirth S, Weigmann B, Ouyang W, Neurath MF and Becker C.. STAT3 links IL-22 signaling in intestinal epithelial cells to mucosal wound healing. J Exp Med. 2009; 206:1465-72.

59. Wittkopf N, Pickert G, Billmeier U, Mahapatro M, Wirtz S, Martini E, Leppkes M, Neurath MF and Becker C. Activation of intestinal epithelial Stat3 orchestrates tissue defense during gastrointestinal infection. PLoS One. 2015;10:e118401.

60. Hutchins AP, Diez D and Miranda-Saavedra D. The IL-10/ STAT3-mediated anti-inflammatory response: recent developments and future challenges. Brief Funct Genomics. 2013;12:489-98.

61. Martínez-Augustin O, Rivero-Gutiérrez B, Mascaraque C and Sánchez de Medina F. Food derived bioactive peptides and intestinal barrier function. Int J Mol Sci. 2014; 15:22857-73.

62. Tanaka T, Kohno H, Suzuki R, Hata K, Sugie S, Niho N, Sakano K, Takahashi M and Wakabayashi K. Dextran 
sodium sulfate strongly promotes colorectal carcinogenesis in $\mathrm{Apc}(\mathrm{Min} /+)$ mice: inflammatory stimuli by dextran sodium sulfate results in development of multiple colonic neoplasms. Int J Cancer. 2006; 118: 25-34.

63. Coulombe G, Langlois A, De Palma G, Langlois MJ, McCarville JL, Gagné-Sanfaçon J, Perreault N, Feng GS, Bercik P, Boudreau F, Verdu EF, Rivard N. SHP-2 Phosphatase Prevents Colonic Inflammation by Controlling Secretory Cell Differentiation and Maintaining HostMicrobiota Homeostasis. J Cell Physiol. 2016; 231:2529-40.

64. Li S, Hsu DDF, Wang H and Feng GS. Dual faces of SH2containing protein-tyrosine phosphatase Shp2/PTPN11 in tumorigenesis. Front Med. 2012; 6: 275-279.

65. Feng GS. Conflicting roles of molecules in hepatocarcinogenesis: paradigm or paradox. Cancer Cell. 2012; 21: 150-154.

66. Fang JY and Richardson BC. The MAPK signalling pathways and colorectal cancer. Lancet Oncol. 2005; 6:322-7.

67. Heuberger J, Kosel F, Qi J, Grossmann KS, Rajewsky K and Birchmeier W. Shp2/MAPK signaling controls goblet/ paneth cell fate decisions in the intestine. Proc Natl Acad Sci U S A. 2014; 111:3472-7.

68. Van der Sluis M, De Koning BA, De Bruijn AC, Velcich A, Meijerink JP, Van Goudoever JB, Büller HA, Dekker J, Van Seuningen I, Renes IB and Einerhand AW. Muc2-deficient mice spontaneously develop colitis, indicating that MUC2 is critical for colonic protection. Gastroenterology. 2006; 131:117-29.

69. Simoneau M, Coulombe G, Vandal G, Vézina A and Rivard N. SHP-1 inhibits $\beta$-catenin function by inducing its degradation and interfering with its association with TATAbinding protein. Cell Signal. 2011; 23:269-79.

70. Bian B, Mongrain S, Cagnol S, Langlois MJ, Boulanger J, Bernatchez G, Carrier JC, Boudreau F and Rivard N. Cathepsin B promotes colorectal tumorigenesis, cell invasion, and metastasis. Mol Carcinog. 2016; 55:671-87.

71. Garneau H, Paquin MC, Carrier JC and Rivard N. E2F4 expression is required for cell cycle progression of normal intestinal crypt cells and colorectal cancer cells. J Cell Physiol. 2009; 221: 350-358.

72. Wang HC, Chiang WF, Huang HH, Shen YY and Chiang HC. Src-homology 2 domain-containing tyrosine phosphatase 2 promotes oral cancer invasion and metastasis. BMC Cancer. 2014; 14: 442.

73. Langlois MJ, Roy SA, Auclair BA, Jones C, Boudreau F, Carrier JC, Rivard N and Perreault N. Epithelial phosphatase and tensin homolog regulates intestinal architecture and secretory cell commitment and acts as a modifier gene in neoplasia. FASEB J. 2009; 23: $1835-1844$.

74. Perreault N, Sackett SD, Katz JP, Furth EE, Kaestner KH. Foxl1 is a mesenchymal Modifier of Min in carcinogenesis of stomach and colon. Genes Dev. 2005; 19:311-5. 